\title{
CALCULATION OF THERMAL \\ NEUTRON FLUXES \\ IN PRIMARY SHIELDS
}

November 1959

CONTRACT AT-11-1-GEN-14

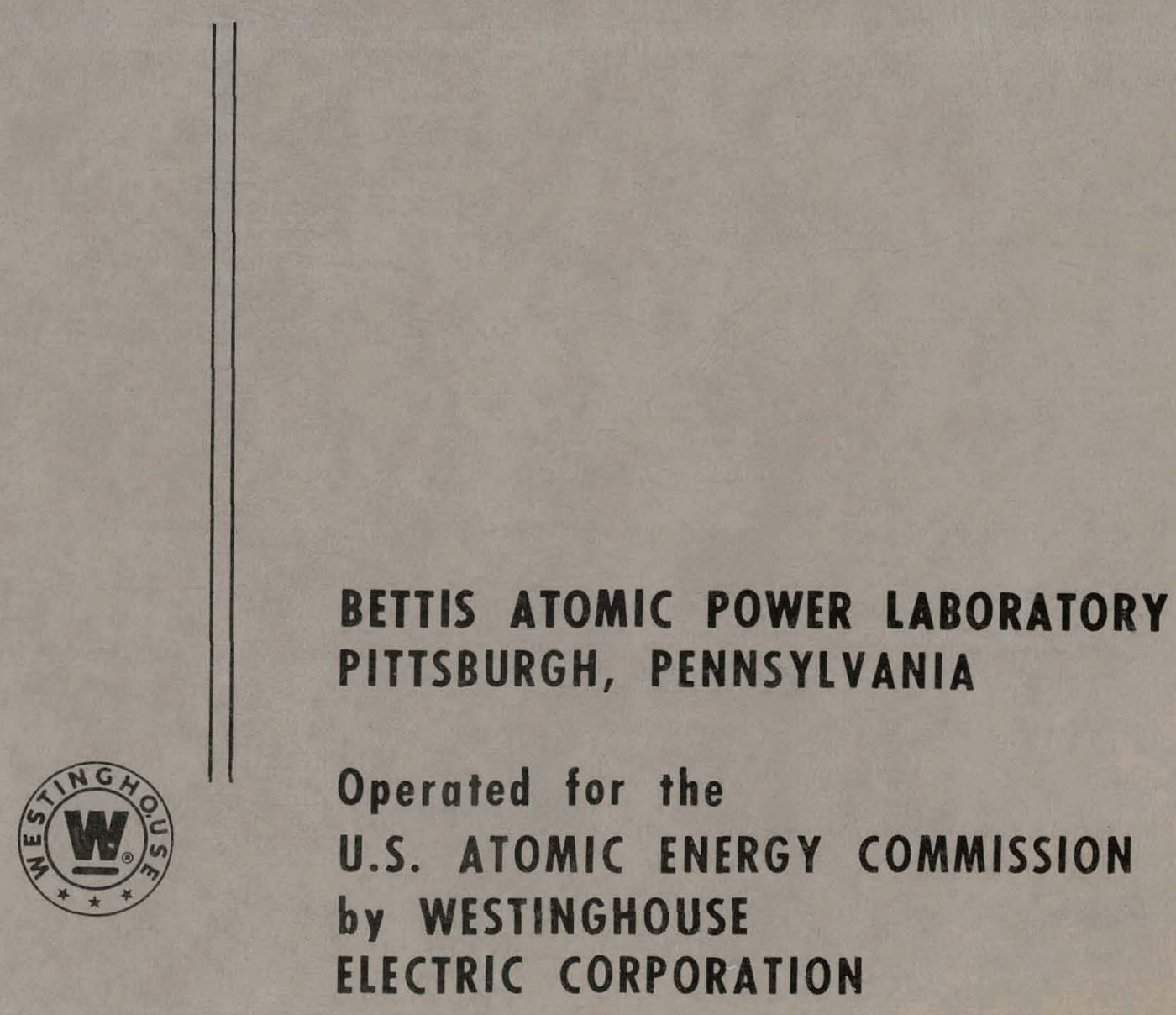




\section{DISCLAIMER}

This report was prepared as an account of work sponsored by an agency of the United States Government. Neither the United States Government nor any agency Thereof, nor any of their employees, makes any warranty, express or implied, or assumes any legal liability or responsibility for the accuracy, completeness, or usefulness of any information, apparatus, product, or process disclosed, or represents that its use would not infringe privately owned rights. Reference herein to any specific commercial product, process, or service by trade name, trademark, manufacturer, or otherwise does not necessarily constitute or imply its endorsement, recommendation, or favoring by the United States Government or any agency thereof. The views and opinions of authors expressed herein do not necessarily state or reflect those of the United States Government or any agency thereof. 


\section{DISCLAIMER}

Portions of this document may be illegible in electronic image products. Images are produced from the best available original document. 


\section{CALCULATION OF THERMAL NEUTRON FLUXES \\ IN PRIMARY SHIELDS}

D. C. Anderson and K. Shure.

November 1959

Contract AT-11-1-GEN-14

Price $\$ 1.25$

Available from the office of Technical Services

Department of Commerce,

Washington $25, \mathrm{D}: \mathrm{C}$.

This document is an interim memorandum prepared primarily for internal reference and does not represent.a final expression of the opinion of Westinghouse. When this memorandum is distributed externally, it is with the express understanding that Westinghouse makes no representation as to completeness, accuracy, or usability of information contained therein.

BETTIS ATOMIC POWER LABORATORY, PITTSBURGH, PA. Operated for the U. S. Atomic Energy Commission by Westinghouse Electric Corporation 
SPECIAL EXTERNAL DISTRIBUTION

Manager, Pittsburgh Naval Reactors Operations Office, AEC Director, Development Division, PNROO

This report was prepared as an account of Government sponsored work, Neither the United States, nor the Commission, nor any person acting on behalf of the Commission:

A. Maises any warranty or representation, expressed or implied, with respect to the accuracy, completeness, or usefulness of the information contained in this report, or that the use of any information, apparatus, method, or process disclosed in this report may not. infringe privately owned rights: or

B. Assumes any liabilities with respect to the use of, or for damages resulting from the use of any information, apparatus, method, or process disclosed in this report.

As used in the above, "person acting on behalf of the Commission" includes any employe or contractor of the Commission, or employe of such contractor, to the extent that such employe or contractor of the Commission, or employe of such contractor prepares, disseminates, or provides access to, any information pursuant to his employment or contract with the Commission, or his employment with such contractor. 
I. INTRODUCTION

II. GENERAL DESCRIPTION OF THE METHOD 3

III. DETAILED DESCRIPTION OF METHOD FOR RADIAL CALCULATIONS 5

IV. POINT ATTENUATION KERNELS FOR THERMAL NEUTRON FLUXES IN WATER 7

A. Determination of Point Kernel at STP 7

B. Temperature Dependence of Point Kernel 13

$\begin{array}{ll}\text { V. COMPARISON WITH EXPERIMENT IN WATER ONLY } & 18\end{array}$

A. Results for the Bulk Shielding Reactor 18

B. Results for the LIDO Reactor · 19

VI. RATIO OF THERMAL NEUTRON FLUX THROUGH A METAL-HYDROGENOUS CONFIGURATION TO THAT IN AN ALI-HYDROGENOUS CONFIGURATION

A. Results for $\mathrm{Fe}-\mathrm{H}_{2} \mathrm{O}$ at the LIDO Reactor 21

B. Results for $\mathrm{Fe}-\mathrm{H}_{2} \mathrm{O}$ at the ORNL Lid Tank Facility . $29^{\circ}$

C. Results for Stainless Steel-Oil at the ORNL Lid
Tank Facility

D. Results for Lead-Oil at the ORNL Lid Tank Facility 32

VII. COMPARISON OF CALCULATION TECHNIQUE WITH EXPERIMENT IN AN IRON-WATER CONFIGURATION 32

VIII. EXTENSION TO TWO DIMENSIONS AND EPITHERMAL FLUXES 35

A. Two Dimensions $\quad 35$

B. Estimallun of Epithermal Fluxes

IX. ADEQUACY OF PIMG SPECTRAL RESULTS $\quad 36$

X. SUMMARY AND CONCLUSIONS

APPENDIX: COMPARISON WITH OTHER PUBLISHED METHODS $\cdot \cdot 38$

ACKNOWLEDGEMENT. $\quad 40$

$\begin{array}{ll}\text { REFERENCES } & 40\end{array}$ 
A method is presented for calculating thermal neutron fluxes in the primary shields of reactor systems which eliminates reliance on mock-up experimental data. A multigroup $\mathrm{P}_{1}$ approach is employed with the spatial dependence of the neutron attenuation adjusted through use of a point source attenuation kernel for a homogeneous hydrogenous medium. Comparison of calculation with experiment is presented.

\section{CALCULATION OF. THERMAL NEUTRON FLUXES}

IN PRIMARY SHIELDS

D. C. Anderson and $K_{0}$ Shure

\section{INTRODUCTION}

An essential requirement in shield design is the ability to estimate the distribution of secondary gamma-ray sources in the reflector, core container and structure, and reactor shield during reactor operation and after shutdown. These gamma rays arise principally from neutron capture during operation and from induced activity after shutdown. Their distribution is dictated to the greatest extent by the thermal neutron flux distribution in the primary shield during reactor operation.

The problem of determining the thermal neutron flux distribution in the shield surrounding a reactor is an imposing one theoretically. The shield in general is large; that is, it provides for a significant attenuation of the neutrons. The shield is also heterogeneous, usually consisting of laminations of water and heavily absorbing metal. Whereas a transport theory calculation such as the moments method can handle large attenuations, it cannot readily cope with inhomogeneities. On the other hand, although diffusion calculations can, deal with heterogeneous media, this calculational technique is inadequate at great penetrations from a source. Thus, up to the present, the shield designer has resorted to semiempirical techniques requiring scale-up of mock-up experimental information in conjunction with diffusion calculations and "removal" theory based on "uncollided flux" calculations to estimate the flux distribution in reactor shields (see Refs 1 and 2 )。 
This report presents a method for calculating the thermal neutron flux distribution in primary shields which eliminates reliance on mock-up experimental data. In summary, a multigroup $\mathrm{P}_{\ell}$ approach is applied to describe the flux distribution in the core and beyond. Microscopic cross sections are used in the $\mathrm{P}_{\ell}$ calculation, including those for inelastic scattering. The model discussed herein is limited to a $P_{1}$ multigroup approximation. Since this description does not adequately describe the neutron attenuation at large distances from the source, a point attenuation kernel for a homogeneous, hydrogenous medium is used to correct the spatial dependence of the $\mathrm{P}_{1}$ results. The attenuation kernel can be obtained either from basic experimental attenuation data or from transport theory calculation such as the moments method, which have been developed for homogeneous media. Thus, the $P_{1}$ model gives dependable information on the perturbation of the flux near and within heavily absorbing portions of the shield (as well as in the core) and a neutron attenuation kernel adjusts the over-all attenuation. Approaches using higher values of $l$ may not require any spatial correction factor.

The procedure admittedly suffers from several inadequacies and deficiencies which will be evident in the description in the report. However, we believe that it represents a significent improvement over present methods and can serve as a basic procedure subject to revisions as they are developed. Comparison of calculation with experiment is presented.

While the studies were in progress which lead to the method given below, several other workers have presented methods of treating aspects of this problem." Cooper et al ( $\operatorname{Kef}^{3} 3$ ) have made a study of' the t'lux distribution in water and through various configurations of iron and water with the LIDO reactor serving as the source. In addition, Cooper has devised a method for calculating flux distributions in reactor shields which reproduces the LIDO experimental results to within about 20\%. Also, Haffner (Ref 4) reports a method for calculating neutron spectra in reactor shields. Discussion concerning these two methods will be found in the Appendix of this report. 
II. GENERAL DESCRIPTION OF THE METHOD

The technique developed below has as its principal goal the calculation of the flux of thermal neutrons. The quantity of interest for our purposes is the "2200 m/sec flux", i.e., the ratio of the thermal activation rate for a $\mathrm{l} / \mathrm{v}$ detector and the macroscopic activation cross section characteristic of the detector at $2200 \mathrm{~m} / \mathrm{sec}(\operatorname{Ref} 5)$. While the use of the thermal neutron flux defined this way readily leads to physical quantities in media characterized by $I / v$ absorption, analytic determination of this specific flux depends on a semiempirical treatment. Specifically, when we have occasion to describe a thermal neutron flux by means of a diffusion equation with a Laplacian $1 / L^{2}$ (see Eq 9, Section IV), the meaning to be attached to this is that there exists an experimental parameter, $L$, which describes the spatial dependence of the $2200 \mathrm{~m} / \mathrm{sec}$ flux. The magnitude of the flux obtained from this type of consideration is normalized by means of the constant $C\left(E_{0}, E_{l}\right)$ appearing in $E q(9)$. The value of this constant is chosen such that the quantities it depends on are compatible with the concept of $2200 \mathrm{~m} / \mathrm{sec}$ flux.

As will be noted in the subsequent discussion, it is convenient, in a limited spatial region, to use multigroup diffusion calculations directly to estimate the flux integrated over the thermal group. These fluxes must be. renormalized to correspond to $2200 \mathrm{~m} / \mathrm{sec}$ fluxes. The appropriate renormalization is obtained by setting the interaction rates for each type of flux equal $\left(\Sigma_{2200} \Phi_{2200}=\Sigma_{\text {th }}^{P_{1}} \stackrel{P_{1 h}}{P_{t h}}\right)$.

Now that our meaning of thermal neutron flux is defined, we present a general description of the method of calculation as it relates to water-metal primary shields specifically. Extension to other shield compositions should be evident from the description presented.

This method uses a $P_{1}$ multigroup diffusion calculation of the flux in the core as well as in the shield. Adequate account for deep penetration from the source of neutrons is accomplished through correction factors. These correction factors, which are applied at distances greater than about $20 \mathrm{~cm}$ from the reactor are obtained from $\mathrm{P}_{1}$ calculations with water surrounding the core and from the

\footnotetext{
Bare foil minus cadmium-covered foil activation rates.
} 
use of point kernels in water. At these distances, the flux can be written as follows :

$$
\Phi_{t h}(r)=\frac{\phi_{W}^{P \cdot K}(r) \phi^{P I M G}(r)}{\phi_{W}^{P I M G}(r)},
$$

where $\Phi_{t h}(r)$ is the thermal neutron flux at a distance $r$ from the core in the actual metal-water configuration, ${ }_{\phi} P I M G(r)$ is the $P_{1}$ multigroup estimate of this quantity, $\Phi_{W}^{P \cdot K} \cdot(r)$ is the thermal neutron flux at a distance $r$ in an all-water
medium surrounding the core,

and $\phi_{W}^{P \perp M G}(r)$ is the $P_{1}$ multigroup estimate of this quantity.

In view of the steps employed in this evaluation, the method can alternately be described as consisting of a calculation of the flux distribution in water surrounding the core with perturbations due to the presence of nonhydrogenous media (iron, etc.). applied to this distribution. These perturbations are determined from one-dimensional multigroup diffusion calculations rather than from empirical information. The ensuing description will refer to the latter interpretation of the method.

In the estimation of the flux distribution in an all-water medium surrounding the core, integration of point attenuation kernels over the spatial distribution of fissions (the core) will yield results applicable beyond $20 \mathrm{~cm}$ but less than $160 \mathrm{~cm}$ from the core edge. In this integration, nonhydrogenous materials of the core may be accounted for in a reasonable manner by using the ORNL determined effective neutron removal cross sections for these materials (Ref 6). The flux distribution in the region extending to $20 \mathrm{~cm}$ beyond the core surface can be obtained directly from one-dimensional multigroup calculations. The region beyond $160 \mathrm{~cm}$ from the core edge can be treated by assuming that the attenuation kernel used up to that point is applicable.

The flux distribution in the water-metal primary shield is then obtained by applying to this all-water distribution the ratio, $\phi^{P I M G}(r) / \phi_{W}{ }^{P I M G}(r)$, obtained from the results of two similar one-dimensional multigroup diffusion calculations. One calculation includes the thermal shields, pressure vessel and shield tank wall while the second calculation replaces the iron with water. 


\section{DETAILED DESCRIPTION OF METHOD FOR RADIAL CALCULATIONS}

We wish to calculate the radial flux distribution from a cylindrical reactor. A description of the core and reflector is obtained. Atom number densities and temperatures are the minimum information required for an estimate to be made of the flux distribution. However, knowledge of the spatial distribution of fissions and thermal neutron constants within the core would result in an improved estimate of the flux distribution. The reflector, thermal shields, pressure vessel wall, and shield tank walls and shield tank internals are specified and located with respect to the core axis. Voids in the actual primary shield may be collapsed without incurring serious error. This step is required since diffusion calculations become unstable in regions of vanishing number densities. A one-dimensional multigroup diffusion calculation using the PIMG code (Ref 7) is performed employing thermal neutron constants obtained from the SOFOCATE code (Ref 8 ). If thermal constants adequate for reactor design are unavailable for the core, a simple approach is to use Maxwellian averaged constants. In all other regions Maxwellian averaged constants are used. The specific form utilized in the PIMG code consists of the 54-group subdivision of the energy interval between $10 \mathrm{Mev}$ and $0.625 \mathrm{ev}$ as given in the MUFT- 4 code (Ref 9) and a single group describing thermal energies. Because of the detailed spectral treatment, groupwise averages of the fast (nonthermal) constants are considered independent of the spectrum and are stored in a library of

"microscopic" nuclear data:specified by the requester. These "microscopic" data are, therefore, more meaningful physically than fast few-group constants obtained from the same nuclear data but averaged over much broader energy intervals. Except for a description of output information of use in shield design given in Sections VIII and IX, the content of the PIMG code is not reported here. A complete description of this code is given in Ret $\%$

The PIMG output includes an eigenvalue, a normalized fission neutron source distribution and the thermal neutron flux distribution. The eigenvalue indicates the total number of fission neutrons generated per second in a unit height of the core. In practice, if control rods are neglected in the core description, the eigenvalue in a typical problem may be somewhere between 1.I and.1.3. This means that the flux obtained is that which is associated with 1.1 to 1.3 fission neutrons distributed over a unit height of the core. An estimate of the absolute flux from an operating core is obtained by multiplying the calculated fluxes by the ratio of neutrons generated per $\mathrm{cm}$ of height 
of the operating core to the PIMG eigenvalue. A similar one-dimensional problem is performed with nonhydrogenous materials external to the core replaced by water at the temperature and density of that with which the material is contiguous. The pointwise ratio of results is to be used as a correction to a flux distribution for pure water that is still to be determined.

The all-water distribution is determined using point attenuation kernels and the geometry of the second PIMG problem, the one in which nonhydrogenous materials external to the core are replaced by water. The outer surface of the pressure vessel wall will be chosen as an arbitrary boundary between an inner high-temperature water region and an outer low-temperature water region. Equations (2) and (3) which are derived in Section IV are exponential representations of attenuation kernels for $2200 \mathrm{~m} / \mathrm{sec}$ fluxes in water at high temperature and low temperature, respectively, from point isotropic fission neutron sources and may, therefore, be utilized to obtain the flux distribution in the artificial geometry of the second PIMG problem.

$$
\begin{aligned}
& \frac{4 \pi r^{2} \Phi_{\text {High T }}(r)}{S}=-117 \mathrm{e}^{-0.2792 \rho^{\prime} r}+59.5 \mathrm{e}^{-0.1907 \rho^{\prime} r} \\
& +2.83 \mathrm{e}^{-0.1117 \rho^{\prime} \mathrm{r}} \quad 450^{\circ} \mathrm{F} \leq \mathrm{T} \leq 650^{\circ} \mathrm{F} \\
& 15 \mathrm{~cm} \leq \rho^{\prime} r \leq 90 \mathrm{~cm}
\end{aligned}
$$

$\frac{4 \pi x^{2} \Phi_{\text {Low } T}(r)}{S}=17.2 e^{-0.1557 \rho^{\prime} r}+0.357 \mathrm{e}^{-0.0951 \rho^{\prime} r}$

$$
\begin{aligned}
& 60^{\circ} \mathrm{F} \leq \mathrm{T} \leq 130^{\circ} \mathrm{F} \\
& 15 \mathrm{~cm} \leq \rho^{\prime} \mathrm{r} \leq 160 \mathrm{~cm}
\end{aligned}
$$

where $\rho^{\prime}$ is the specific gravity of water. Specifically, in the hightemperature region, Eq (2) is integrated over the spatial distribution of fission neutron sources. This integration can be approximated by means of the formulae given in Ref 1 or by use of applicable machine codes. For right circular cylindrical reactor cores, the SPIC-1 code (Ref 10) can be used and has the advantage of taking into account the axial as well as radial source density distribution in the core. The results of this calculation are employed beyond about $20 \mathrm{~cm}$ from the core edge. . In the. low-temperature region, a similar' integration is performed using Eq (3). In using these equations, attenuation by. regions of different density is accounted for by adjusting the appropriate 
attenuation coefficients in the equations through the factor $\rho$. At the boundary $\left(r_{b}\right)$, of media of differing temperature, the results obtained using. Eq (2) will exceed those obtained using Eq (3) by some value $\triangle$ given in Eq (4).

$$
\Delta=\phi_{\text {High } T}\left(r_{b}\right)-\phi_{\text {Low } T}\left(r_{b}\right)
$$

The corrected flux in the outer low-temperature region $\left(r>r_{b}\right)$ is taken to be

$$
\phi_{W}^{P_{0} K_{0}}(r)=\phi_{\text {Low } T}(r)+\Delta e^{-\frac{\left(r-r_{b}\right)}{L}}
$$

where $L$ is the diffusion length of thermal neutrons in low-temperature water $(\sim 2.8 \mathrm{~cm})$. Thus the pure water curve has been generated.

The ratio of results obtained from the two PIMG problems is applied pointby-point to the pure water curve.

\section{POINT ATTENUATION KERNELS FOR THERMAL NEUTRON FLUXES IN WATER}

\section{A. Determination of Point Kernel at STP}

We now present a derivation of a point kernel for the flux from a point fission neutron source in water at STP. Certaine and Aronson (Ref 1i) have calculated by the moments method the spatial dependence of the neutron flux at $2.03 \mathrm{ev}$ from a point fission source in water at STP. These results will be used in the derivation. Figure 6-1.3 of Ref 12: presents a comparison between these calculated results and those obtained from experiment (Ref 13). The agreement in shape beyond $10 \mathrm{~cm}$ is good.

It is assumed that the epithermal neutron flux, $\psi(E, r)$, that exists in an infinite water medium containing a point fission source is a separable function of position and energy; that is,

$$
\psi(E, r)=\Psi\left(E_{1}, r\right) f\left(E, E_{1}\right)
$$

where $\Psi\left(E_{1}, r\right)$ is the moments method flux at $E_{1}=2.03 \mathrm{ev}$, and $f\left(E_{1} E_{1}\right)$ is the energy dependent function. It is also assumed that an energy, $E_{0}$, exists which defines the energy boundary between thermal and epithermal neutrons. Now a source of nascent thermal neutrons $\left(E<E_{0}\right)$ can be obtained if we define $\Sigma\left(E^{\prime} \rightarrow E\right)$ as the probability of a neutron being scattered from $E^{\prime}$ to $E$. 


$$
\begin{aligned}
S_{\operatorname{th}}\left(E, E_{0}, r\right) & =\int_{E_{0}}^{\infty} \psi\left(E^{\prime}, r\right) \Sigma\left(E^{\prime} \rightarrow E\right) d E^{\prime} \ldots \\
& =\Psi\left(E_{I}, r\right) \int_{E_{0}}^{\infty} f\left(E^{\prime}, E_{1}\right) \Sigma\left(E^{\prime}-E\right) d E^{\prime} \\
& =\Psi\left(E_{1}, r\right) k\left(E_{O}, E_{I}, E\right) \quad E<E_{0} .
\end{aligned}
$$

The total source, commonly called the slowing-down density for thermal neutrons, is obtained as

$S(r)=\int_{0}^{E_{0}} S_{t h}\left(E, E_{0}, r\right) d E=\Psi\left(E_{1}, r\right) \int_{0}^{E_{0}} k\left(E_{0}, E_{I}, E\right) d E=\Psi\left(E_{1}, r\right) K\left(E_{0}, E_{l}\right)$.

Thus the source can be taken to be proportional to the moments method results at $2.03 \mathrm{ev}$. One could have initially made this assumption. The reasoning presented indicates how $\mathrm{K}\left(\mathrm{E}_{0}, \mathrm{E}_{1}\right)$ can be derived from physical quantities such as scattering cross sections. We choose to arrive at a quantity related to $K\left(E_{0}, E_{l}\right)$ in the following manner.

Thermal neutrons diffuse in water according to the following equation

$$
\left(\nabla^{2}-i / L^{2}\right) \phi_{t h}(r)=-\Psi\left(E_{l}, r\right) C\left(E_{0}, E_{l}\right)
$$

where $L$ is the thermal neutron diffusion length in water. The solution to this equation is

$$
\Phi_{t h}(r)=\int_{a l l \text { Epace }} \frac{C\left(E_{0}, E_{I}\right) \Psi\left(E_{I}, \underline{r}^{\prime}\right) e^{-\frac{\left|\underline{r}-\underline{r}^{\prime}\right|}{I}}}{4 \pi\left|\underline{r}-\underline{r}^{\prime}\right|} d V^{\prime}
$$

The zero-th moment of a radial function, $f(r)$; in spherical coordinates is given as

$$
f^{(0)}=\int_{0}^{\infty} 4 \pi r^{2} f(r) d r
$$


The relation between the zero-th moments of the thermal neutron and $2.03 \mathrm{ev}$ fluxes, from Eq (10), is

$$
\Phi_{\text {th }}^{(0)}=C\left(E_{0}, E_{1}\right) \Psi^{(0)}\left(E_{1}\right) L^{2}
$$

To determine the value of $C\left(E_{O}, E_{1}\right)$, we write an equation which is a statement of the conservation of neutrons, namely

$$
\Sigma_{a}^{\operatorname{th}}{ }_{t h}^{(0)}+\int_{E_{0}}^{\infty} \Sigma_{a}(E) \phi(0)(E) d E=1
$$

where $\Sigma_{a}(E)$ is the macroscopic absorption cross section as a function of energy. This equation states that for a source emitting one neutron per unit time in an infinite homogeneous medium, one neutron is captured per unit time. The capture of the neutron as a function of energy is written in this equation, remembering that $\mathrm{E}_{\mathrm{O}}$ defined the energy boundary between thermal and epithermal neutrons.

The integral can be evaluated by making two reasonable assumptions. The first assumption is that the absorption cross section for water varies as $I / v$; that is,

$$
E^{1 / 2} \Sigma_{a}(E)=E_{1}^{1 / 2} \Sigma_{a}\left(E_{1}\right)
$$

The second assumption is that the zero-th moment of the epithermal neutron flux varies as $I / E$; that is,

$$
E \Psi^{(0)}(E)=E_{1} \Psi^{(0)}\left(E_{l}\right)
$$

This assumption has been shown to be very good over wide energy ranges in water.

An evaluation of the integral yields

$$
\int_{E_{0}}^{\infty} E_{l}^{l / 2} \Sigma_{a}\left(E_{1}\right) E_{1} \Psi^{(0)}\left(E_{1}\right) E^{-3 / 2} d E=2 E_{1} \Psi^{(0)}\left(E_{1}\right) \Sigma_{a}\left(E_{0}\right)
$$


We now rewrite Eq (12):

$$
\Sigma_{a}^{\text {th }} \phi_{t h}^{(0)}+2 E_{I} \Psi^{(0)}\left(E_{1}\right) \Sigma_{a}\left(E_{0}\right)=1
$$

Into this equation, we substitute the value of $\phi_{t h}^{(0)}$ from $\mathrm{Eq}(11)$ and solve for $C\left(E_{0}, E_{1}\right)$ :

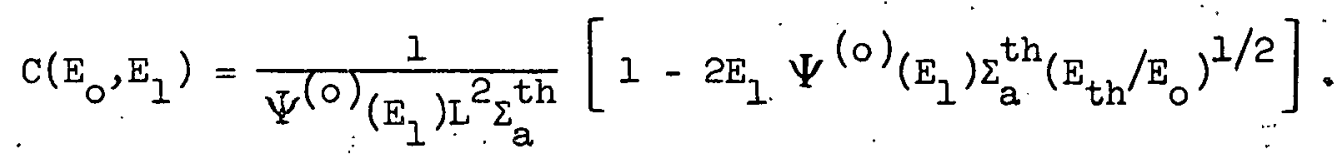

It is easily shown that the second term in the brackets is small compared with unity. Therefōre $C\left(E_{0}, E_{1}\right)$ is insensitive to the exact choice of $E_{0}$. We have evaluated $C\left(E_{0}, E_{1}\right)$ using the following information:

$$
\begin{aligned}
& \Psi(0)(2.03 \mathrm{ev})=3.67 \times 10^{5} \mathrm{n}-\mathrm{cm} / \mathrm{sec}-\mathrm{Mev}(\text { Ref II) } \\
& \dot{\mathrm{L}}=2.72 \mathrm{~cm} . \\
& \Sigma_{\mathrm{a}}^{\mathrm{th}}=0.0221 \mathrm{~cm}^{-1} \\
& E_{1}=2.03 \mathrm{ev} \\
& E_{\text {th }}=0.025 \mathrm{ev} \\
& E_{0}=0.625 \mathrm{ev} .
\end{aligned}
$$

Thus $C\left(E_{U}, E_{I}\right)$ is $1.66 \times 10^{=5} \mathrm{Mev} / \mathrm{cm}^{2}$. The second term in the bracket.s is . $6.6 \times 10^{-3}$.

Having determined $\mathrm{C}\left(\mathrm{E}_{0}, \mathrm{E}_{1}\right)$, we have evaluated $\mathrm{Eq}$ (10) by the method described in section IV-B and the results are shown in Fig. $I$ and Table $I$ where they are compared with results derived from experiment. 
TABLE I

Comparison of Semianalytical and Experimental Kernels $4 \pi r^{2} \phi(r)$ (Thermal Neutron/Fission Neutron)

\begin{tabular}{ccccc}
\hline $\mathrm{r}$ & Calculation & & Experiment & Calculation/Experiment \\
$20 \mathrm{~cm}$ & $9.26 \times 10^{-1}$ & & $8.2 \times 10^{-1}$ & 1.13 \\
$40 \mathrm{~cm}$ & $4.25 \times 10^{-2}$ & $4.2 \times 10^{-2}$ & 1.01 \\
$60 \mathrm{~cm}$ & $3.10 \times 10^{-3}$ & $3.1 \times 10^{-3}$ & 1.00 \\
$80 \mathrm{~cm}$ & $3.20 \times 10^{-4}$ & $2.8 \times 10^{-4}$ & 1.14 \\
$90 \mathrm{~cm}$ & $1.10 \times 10^{-4}$ & $8.9 \times 10^{-5}$ & 1.24 \\
\hline
\end{tabular}

We have also directly converted to point geometry out to $120 \mathrm{~cm}$ of water the ORNL Lid Tank $2200 \mathrm{~m} / \mathrm{sec}$ flux data obtained with the new source plate (Ref 14). The results seen in Fig。 1 compare favorably beyond $20 \mathrm{~cm}$ with that reported by Trubey et al (Ref 15). We have added Trubey's results to our values in the region beyond $1.20 \mathrm{~cm}$. The following equation is a representation of this point kernel to within $15 \%$ in the range of $\mathrm{r}$ from $15 \mathrm{~cm}$ to $160 \mathrm{~cm}$ of water.

$$
\frac{4 \pi r^{2} \Phi_{L_{O W} T}(r)}{S}=17.2 e^{-0.1557 r}+0.357 \mathrm{e}^{-0.0951 r}
$$

where $\Phi_{\text {Low } T}(r)$ is the flux $\left(\mathrm{n} / \mathrm{cm}^{2}-\mathrm{sec}\right)$ at a distance $\mathrm{r} \mathrm{cm}$ in water $\left(\mathrm{T}=2930 \mathrm{~K}, \rho=1 \mathrm{gm} / \mathrm{cm}^{3}\right)$ from a point isotropic source emitting $\mathrm{S}$ fission neutrons per second.

It is seen that the theoretically derived point kernel agrees favorably in shape with the experimentally derived point kernel and is of the order of $10 \%$ greater in magnitude. We have chosen to use the experimentally determined thermal neutron point kernel for subsequent low temperature calculations because the information available extends to deeper penetrations than that available from the theoretical development.

It is well to summarize the preceding arguments at this point and iterate the nature and extent of the assumptions required. We have defined a thermal neutron flux, the $2200 \mathrm{~m} / \mathrm{sec} \mathrm{flux}$, in Section II; Equations (9) and (12) were then used to calculate this quantity. Equation (12) follows from this flux definition and the conservation condition without any assumptions. The verity of the left-hand side of $\mathrm{Eq}$ (9) follows from a) the definition of flux and 


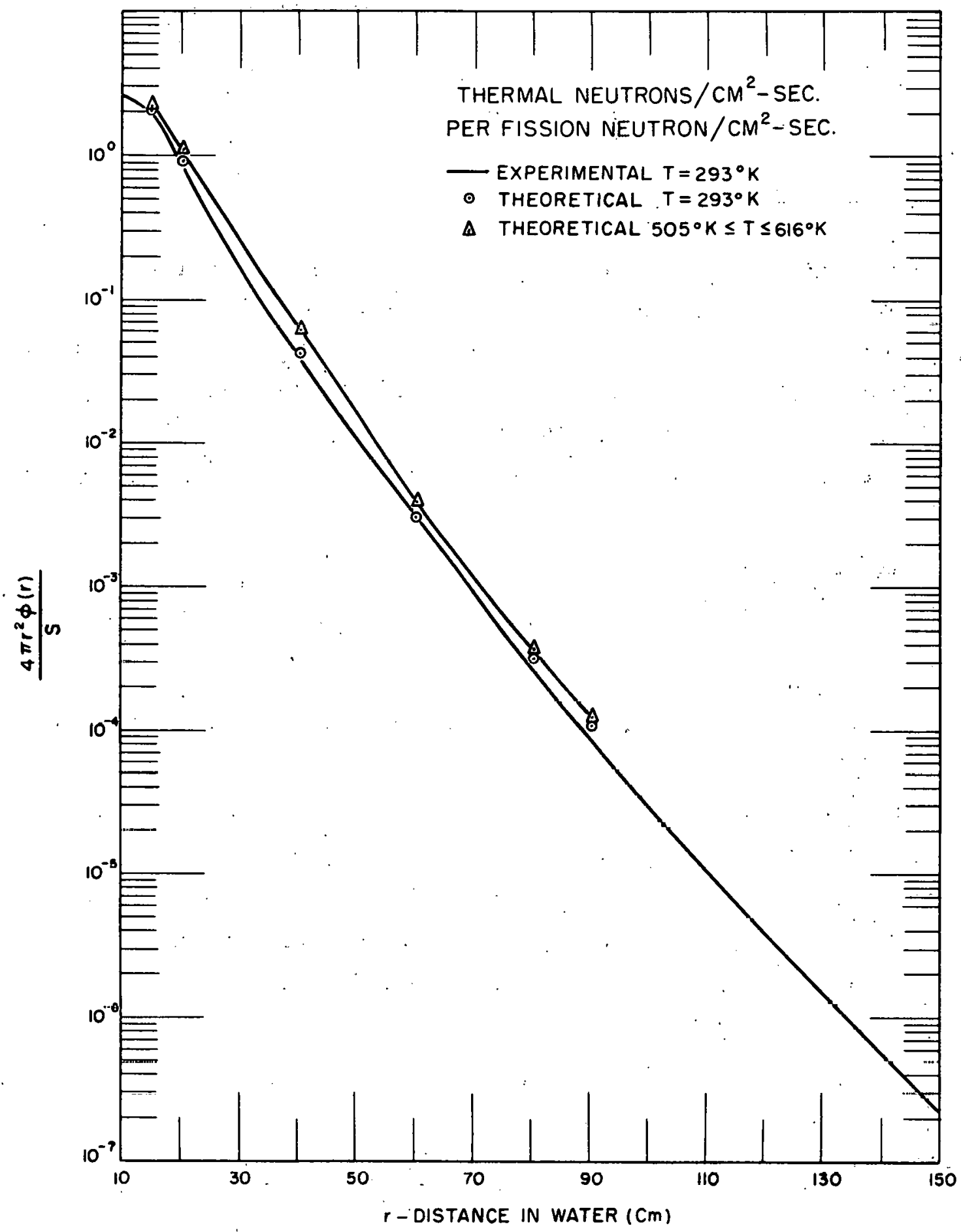

Figure 1 
b) experimental evidence as to the existence of a unique $I$ (at a given temperature) which characterizes this flux. That the source shape may be taken to be that of the spectral flux at an epithermal energy $E_{1}$ follows from an assumption of spatial and spectral separability in the epithermal region. Finally, the exact-determination of the constant $\mathrm{C}$ with which Eq (9) was solved to obtain the normalized $2200 \mathrm{~m} / \mathrm{sec}$ flux depends rather insensitively on the assumptions of an upper boundary, $E_{0}$, to the thermal region, and the $\mathrm{E}^{-1}$ and $\mathrm{E}^{-1 / 2}$ spectral dependences for the zero-th moment of the epithermal flux and the absorption cross section for water, respectively. The validity of the kernel representing the flux from a point isotropic source calculated according to the technique of this section thus depends upon four assumptions: spatial and spectral separability, $E^{-1}$ dependence of the epithermal zero-th moment, $E^{-1 / 2}$ dependence of the absorption cross section for water and the existence of an upper limit to the thermal region, $E_{O^{\circ}}$ The most crucial of these, separability, has been verified over a restricted spectral range by quasi-rigorous arguments。

\section{B. Temperature Dependence of Point Kernel}

Equation $\left(3^{\prime}\right)$ and the point kernel that it fits can be used to calculate the flux in water at temperature and density conditions similar to those in the Lid Tank. A simple change in the water density conditions without any change in the temperature (if this were possible) could be effected by adjusting the attenuation coefficients $\left(0.1557 \mathrm{~cm}^{-1}\right.$ and $\left.0.0951 \mathrm{~cm}^{-1}\right)$ in $\mathrm{Eq}\left(3^{\prime}\right)$ by the density change. A change in temperature may not necessarily be accounted for by a similar simple procedure. For calculations of the thermal neutron flux in water at reactor operating temperatures and densities, a different point kernel is neçessary.

We can derive a point kernel for water at an arbitrary temperature and density. The spatial distribution of the "indium resonance" flux from a point fission neutron source in water was assumed to depend.on the water density but not on the temperature. This distribistion was fitted by the following equation:

$$
\begin{aligned}
& 4 \pi r^{2} \Psi\left(E_{1}, r\right)=6\left(e^{-k_{1} r}+A e^{-k_{2} r}\right) \\
& 15 \mathrm{~cm} \leq \mathrm{r} \leq 90 \mathrm{~cm} \text {. }
\end{aligned}
$$

It is now possibie to evaluate the thermal neutron flux distribution given by $\mathrm{Eq}$ (i0) if the analytic representation of $\mathrm{Eq}$ (15) is used in the integrand. 
Expanding the diffusion kernel in spherical waves, we ultimately obtain Eq (16):

$$
\begin{aligned}
4 \pi r^{2} \Phi(r)=\frac{\delta L r}{2} & C\left(E_{0}, E_{1}\right)\left\{e^{r / L}\left[E_{1}\left\{\left(k_{1}+\frac{I}{L}\right) r\right\}+A E_{1}\left\{\left(k_{2}+\frac{I}{L}\right) r\right\}\right]\right. \\
& \left.-e^{-r / L}\left[E_{1}\left\{\left(k_{1}-\frac{I}{L}\right) r\right\}+A E_{1}\left\{\left(k_{2}-\frac{I}{L}\right) r\right\}\right]\right\}
\end{aligned}
$$

where

$$
\begin{aligned}
& \text { I is the thermal neutron diffusion length, } \\
& \mathrm{E}_{1}(\mathrm{x}) \text { is the exponential integral, } \\
& \mathrm{C}\left(\mathrm{E}_{0}, \mathrm{E}_{1}\right) \text { is defined in section IV-A. }
\end{aligned}
$$

The value of $L$ at varying temperatures is obtained using the output of the SOFOCATE code. Equation (16) has been evaluated for the several conditions found in lable II at six penetration distances in water. It is to be noted that over the wide temperature range investigated represented in Cases 1,2 , and 3 , the numerical results are the same to within about $30 \%$ for the most part. In particular, it should be noted that the dependence of flux on temperature for high temperatures is even smaller (less than $16 \%$ for a $200 \mathrm{~F}^{\circ}$ change). This behavior could have been anticipated if the $E_{1}$ functions in Eq (16) are expanded in their asymptotic series and only the first term in each case is retained. The result is given in Eq (17)。

$4 \pi r^{2} \phi(r) \approx \frac{\delta r^{2}}{\Psi^{(0)_{\Sigma} t h}}\left[\frac{e_{a}^{-k_{1} r}}{\left[1-\left(I k_{1}\right)^{2}\right] r^{2}-L^{2}\left[2 k_{1} r+1\right]}+\frac{A e^{-k_{2} r}}{\left[1-\left(L k_{2}\right)^{2}\right] r^{2}-I^{2}\left[2 k_{2} r+1\right]}\right]$

Equation (17) suggests, at least for large penetrations, that the dependence of $\phi(r)$ on the thermal diffusion length and through it on the temperature is small provided $\mathrm{k}_{1} \mathrm{~L}$ and $\mathrm{k}_{2} \mathrm{~L}$ are small compared to unity. Thus, the temperature dependence appears to a first approximation in its effect on the density of the water medium by changes in the values of $k_{1}$ and $k_{2}$.

A smooth curve through the average values listed in Table TT. for Cases 2 and 3 is plotted in Fig。 $I$ and has been fitted by the following exponential representation: 
TABLE II

\begin{tabular}{|c|c|c|c|c|c|c|c|c|c|}
\hline \multirow[b]{2}{*}{ Case } & \multirow[b]{2}{*}{$\underline{T^{\circ} \mathrm{K}}$} & \multirow[b]{2}{*}{$\underline{T^{\circ} F}$} & \multirow[b]{2}{*}{ Density $\mathrm{gm} / \mathrm{cm}^{3}$} & \multicolumn{4}{|c|}{$\mathrm{rcm}$} & \multicolumn{2}{|l|}{$\therefore \quad}$. \\
\hline & & & & 15 & 20 & 40 & 60 & 80 & 90 \\
\hline 1 & 293 & 68 & 1.0 & $2.07 \times 10^{0}$ & $9.26 \times 10^{-1}$ & $4.25 \times 10^{-2}$ & $3.10 \times 10^{-3}$ & $3.20 \times 10^{-4}$ & $1.10 \times 10^{-4}$ \\
\hline 2 & 505 & 450 & 1.0 & $2.22 \times 10^{0}$ & $1.15 \times 10^{0}$ & $5.66 \times 10^{-2}$ & $3.68 \times 10^{-3}$ & $3.58 \times 10^{-4}$ & $1.22 \times 10^{-4}$ \\
\hline $2 a$ & & & 1.0 & $2.16 \times 10^{0}$ & $1.18 \times 10^{0}$ & $5.98 \times 10^{-2}$ & $4.11 \times 10^{-3}$ & $3.87 \times 10^{-4}$ & $1.24 \times 10^{-4}$ \\
\hline 3 & 616 & 650 & 1.0 & $2.10 \times 10^{0}$ & $1.18 \times 10^{0}$ & $6.54 \times 10^{-2}$ & $4.10 \times 10^{-3}$ & $3.82 \times 10^{-4}$ & $1.29 \times 10^{-4}$ \\
\hline 4 & 561 & 550 & 0.8 & $2.91 \times 10^{0}$ & $1.95 \times 10^{0}$ & $2.02 \times 10^{-1}$ & $1.92 \times 10^{-2}$ & $2.36 \times 10^{-3}$ & $9.13 \times 10^{-4}$ \\
\hline $4 a$ & 561 & 550 & 0.8 & $2.67 \times 10^{0}$ & $1.95 \times 10^{0}$ & $1.97 \times 10^{-1}$ & $1.94 \times 10^{-2}$ & $2.52 \times 10^{-3}$ & $9.75 \times 10^{-4}$ \\
\hline
\end{tabular}


$\frac{4 \pi r^{2} \phi_{\text {High } T}(r)}{S}=-117 e^{-0.2792 r}+59.5 e^{-0.1907 r}+2.83 e^{-0.1117 r}$

Case 2a presents the results using this equation, exhibiting a deviation of less than 10\% from the values for Cases 2 and 3 . Case $4 a$ presents the results using Eq (2) with a value of $\rho^{\prime}=0.8$. Examining the results of Cases 4 and 2 or 3 indicates a density dependence of the point kernel with differences increasing with penetration. An examination of Cases 4 and $4 a$ indicates that a change in density can be adequately accounted for by adjusting the exponential attenuation coefficients.

The following is presented as a check on the derived temperature variation of the point kernel and as a substantiation to the assumption that the $\Delta$ given in Section III indeed falls off with a relaxation length approximating the diffusion length of thermal neutrons in cold water. A cylindrical core at operating temperature and density is surrounded by a $25 \mathrm{~cm}$ reflector of high-temperature water and this is in turn surrounded by low-temperature water. Figure 2 presents PIMG f'lux results in the region from 15 to $42 \mathrm{~cm}$ from the core edge. In this region, diffusion results and point kernel results are presumed to be believable. Also shown are the flux results obtained in the high-temperature region using a modification of $\mathrm{Eq}\left(2^{\prime}\right)^{\mathrm{k}}$ and in the lowtemperature region using $\mathrm{Eq}\left(3^{\prime}\right)$ with the results in the high-temperature water region normalized to the PIMG calculations. The asymptotic agreement between the low-temperature kernel results and those of PIMr is gnod. Furthermore, if the difference between the PIMG and the low-temperature point kernel results in the low-temperature region is plotted as a function of distance (as is also seen in Fig. 2), the relaxation length found is $3.2 \mathrm{~cm}$. This is in reasonable agreement with the thermal neutron diffusion length in the low-temperature region.

\footnotetext{
F'or this calculation, the results obtained using Eq (2') were multiplied by the ratio of the $2200 \mathrm{~m} / \mathrm{sec}$ macroscopic cross section for water to the value obtained from the SOFOCATE code. This factor is necessary since the diffusion parameters utilized in PIMG are not consistent with the "2200 m/ $/ \mathrm{sec}^{\prime \prime}$ flux definition.
} 


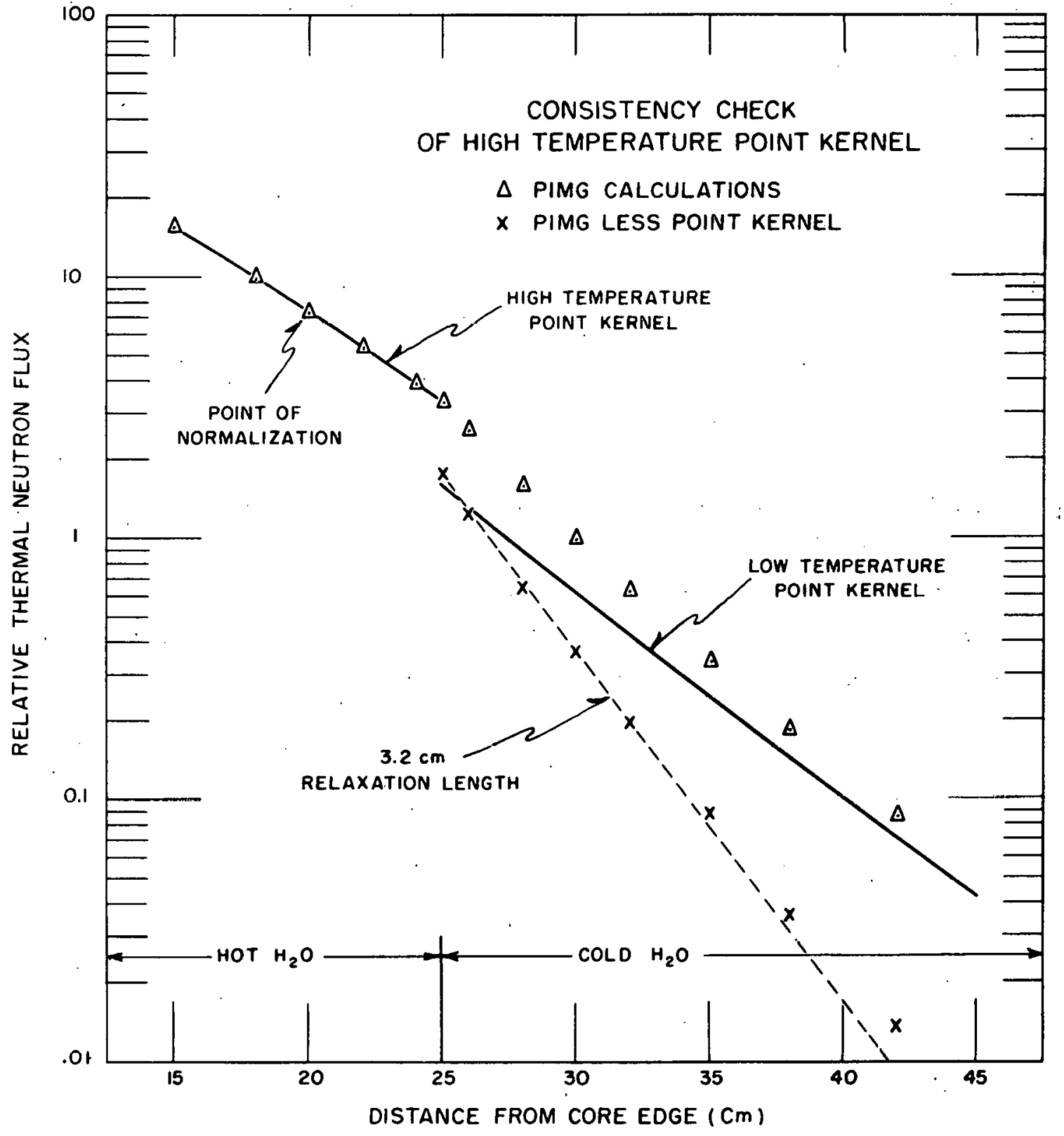

Figure 2 
V. COMPARISON WITH EXPERIMENT IN WATER ONLY

\section{A. Results for the Bulk Shielding Reactor}

We now present a comparison of calculation using the low-temperature : point attenuation kernel with experimental results in which the source density distribution is fairly well known. Since the kernel is based on experiment, these comparisons are performed only to check the application of the procedure to a reactor source.

The flux distribution has been measured along one of the axes of the Bulk Shielding Reactor (Ref 16). These fluxes have been adjusted so that they correspond to the neutron densities times $2200 \mathrm{~m} / \mathrm{sec}$ and the results are seen in Table III. The reactor configuration and the power density distribution in the core have been reported in Ref.17. We have integrated Eq (3) over the core using 1.31 barns/atom for the Al effective removal cross section. The integration was carried out over 25 of the 28 elements in the BSR core; which elements develop $90.6 \%$ of the power in the core. When the last three elements are included, the calculated results are not increased by as much as 1\%. Each element was divided into ten equal volume elements each 2.4 in. high $x 3$ in. $x 3$ in. Each volume source was replaced by a point source located in the center of the volume element whose strength is equal to the total source in the element. The axial variation of the power density distribution was assumed to be separable from the variation in the other two dimensions and was derived from averages over all elements of the axial fall-off presented in Ref 17 assuming symmetry about the horizontal core center plane. Table IV presents the relative point source strength axial variation about the core center plane. 


\begin{tabular}{|c|c|c|c|}
\hline \multirow[b]{2}{*}{$\begin{array}{l}\text { Distance } \\
\text { from Core } \\
\end{array}$} & \multicolumn{3}{|c|}{$\frac{2200 \mathrm{~m} / \mathrm{sec} F l u x}{\text { Watt in Reactor }}\left(\frac{\mathrm{n} / \mathrm{cm}^{2}-\mathrm{sec}}{\text { Watt }}\right)$} \\
\hline & Experiment & Calculation & $\frac{\text { Calculation }}{\text { Experiment }}$ \\
\hline $\begin{array}{r}20 \mathrm{~cm} \\
60 \mathrm{~cm} \\
100 \mathrm{~cm} \\
150 \mathrm{~cm}\end{array}$ & $\begin{array}{l}4.17 \times 10^{5} \\
4.80 \times 10^{2} \\
2.75 \times 10^{-2} \\
1.07 \times 10^{-2}\end{array}$ & $\begin{array}{l}4.33 \times 10^{5} \\
4.75 \times 10^{2} \\
2.82 \times 10^{-2} \\
1.14 \times 10^{-2}\end{array}$ & $\begin{array}{l}1.04 \\
0.99 \\
1.03 \\
1.07\end{array}$ \\
\hline
\end{tabular}

TABLE IV

\begin{tabular}{ll}
\hline & \\
\pm 1.2 in. & 0.980 \\
\pm 3.6 in. & 0.914 \\
\pm 6.0 in. & 0.826 \\
\pm 8.4 in. & 0.739 \\
\pm 10.8 in. & 0.652 \\
\hline
\end{tabular}

In Fig. 3 there is seen the variation of the source density distribution in the volume elements adjacent to the core center plane used in these calcu-: lations. The results of the integration are also seen in Table III as well as the ratio of the calculated to experimental results. The results of integrating the experimentally derived point kernel over the BSR source distribution are in excellent agreement with the experimental values in the region from $20 \mathrm{~cm}$ to $150 \mathrm{~cm}$ of water.

\section{B. Results for the LIDO Reactor}

Cooper has integrated the Albert-Welton point kernel given in Eq (13), using a multipoint source technique over the LIDO core employing an oxygen removal cross section of 0.91 barns and an aluminum removal cross section of I.31 barns. These removal flux results when multiplied by a factor of "about 54 " are reported to reproduce the magnitude and shape of the $2200 \mathrm{~m} / \mathrm{sec}$

\footnotetext{
In Ref 3, Cooper reports that the factor should be $35 \pm 3.5$. However, as a result of a recent power recalibration of the LIDO reactor, Cooper (Ref. 18) reports a factor of the order of 54 .
} 
PLAN VIEW OF BSR INDICATING RELATIVE SOURCE DENSITY DISTRIBUTION IN HORIZONTAL CORE CENTER PLANE

LOCAL SOURCE DENSITY FOR REACTOR POWER OF I WATT $\left\{10^{6}\right.$ fission neutrons $/ \mathrm{cm}^{3}-\mathrm{sec}$ ?

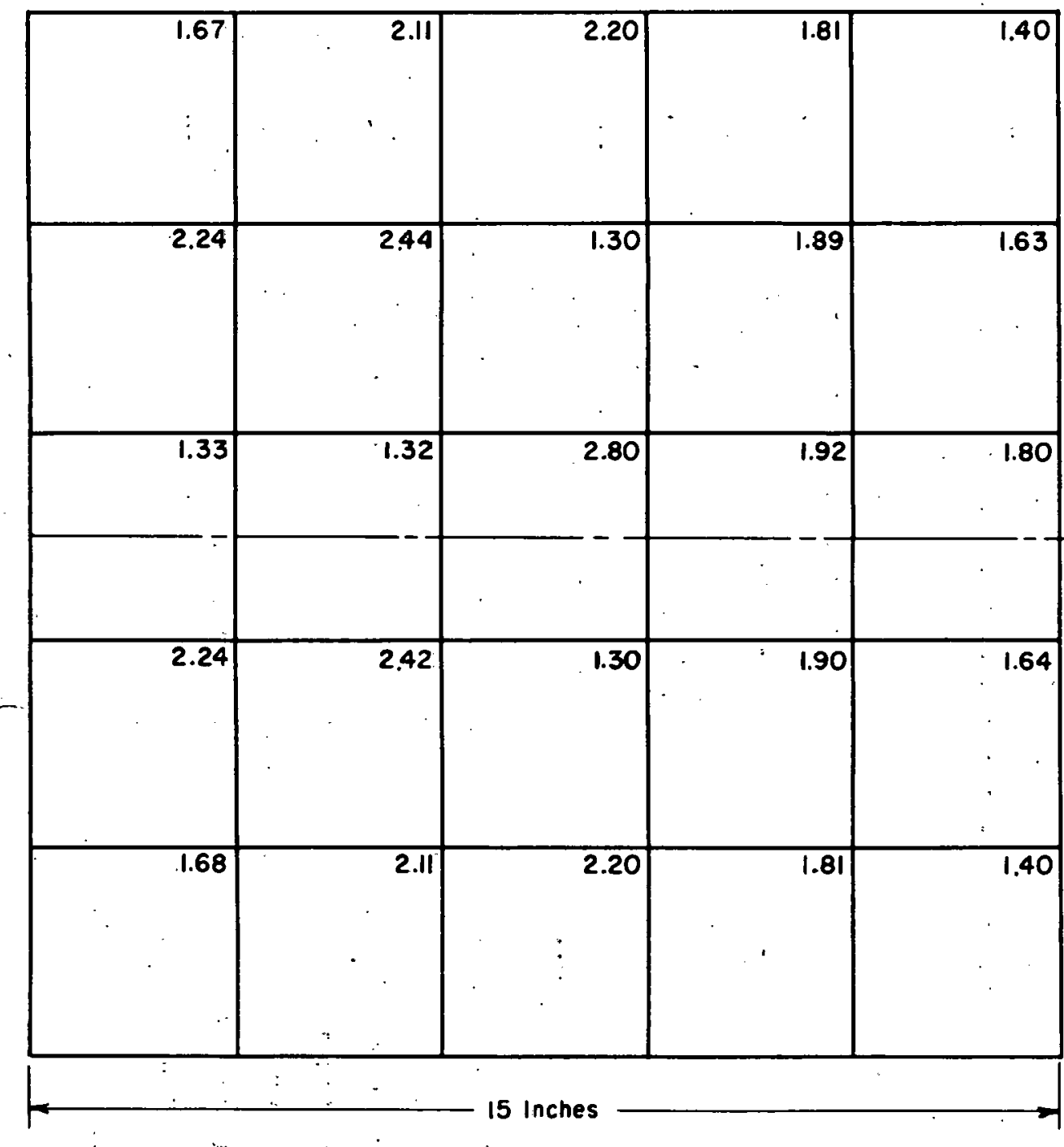

MEASUREMENTS TAKEN ALONG THIS AXIS 
flux observed in the Panel C Facility water and in the LIDO pool.

$$
\frac{4 \pi Z^{2} \phi(Z)}{S}=5.4(Z-T)^{0.29} e^{-0.928(Z-T)^{0.58}-(Z-T) \Sigma_{0}-\sum_{i} \Sigma_{r_{i}} t_{i}} \text {, }
$$

where $\Phi(z)$ is the removal flux $\left(\mathrm{n} / \mathrm{cm}^{2}-\mathrm{sec}\right)$ in water $\mathrm{z} \mathrm{cm}$ from a point source having a strength of one fission per second. The oxygen removal cross section is denoted by $\Sigma_{0}$ and $t_{i}$ is the thickness of material $i$ other than water having removal cross sections $\Sigma_{r_{i}}$ and a total thickness $\left(T=\sum_{i} t_{i}\right)$.

We have evaluated $\mathrm{Eq}$ (18) for water alone using an oxygen removal cross section of 0.91 barns over the range of $\mathrm{Z}$ from $20 \mathrm{~cm}$ to $100 \mathrm{~cm}$ of water. The pointwise ratio of the flux calculated by Eq (3) to that given in Eq (18), when the source strength in Eq (18) is one fission neutron per second, is quite independent of position and has the value, $54.7 \pm 1.9$. It is clear then, in view of the relationship observed by Cooper between the Albert-Welton kernel and the measured flux in the IIDO reactor pool, and that presented above between the Albert-Welton and thermal flux kernels; that the thermal flux kernel would have predicted the thermal fluxes in the LIDO pool and the Panel C Facility adequately.

The comparisons reported above constitute proof that the Green's function approach is numerically valid in the important case of a reactor source surrounded by water, within the restricted ranges noted above.

VI. RATIO OF THERMAL NEUTRON FLUX THROUGH A METAL-HYDROGENOUS CONFIGURATION TO THAT IN AN ALL-HYDROGENOUS CONFIGURATION

A. Results for $\mathrm{Fe}-\mathrm{H}_{2} \mathrm{O}$ at the LIDO Reactor

The procedure described obtains the ratio of the flux in an $\mathrm{Fe}-\mathrm{H}_{2} \mathrm{O}$ configuration to that in an all-water configuration from PIMG calculations. We will now compare the result of this procedure with actual experimental ratios observed by Cooper in the Panel C Facility of the LIDO reactor (Ref 3 ).

PIMG problems were run utilizing the assumed geometric configuration given in Fig. 4 with the iron slabs of the several experiments replaced by concentric annular cylinders located at the same material distance from the core centerline (the void in the actual experiment being collapsed). In Figs. 5 to 10 are 
LIDO PANEL C FACILITY

ACTUAL PLAN VIEW

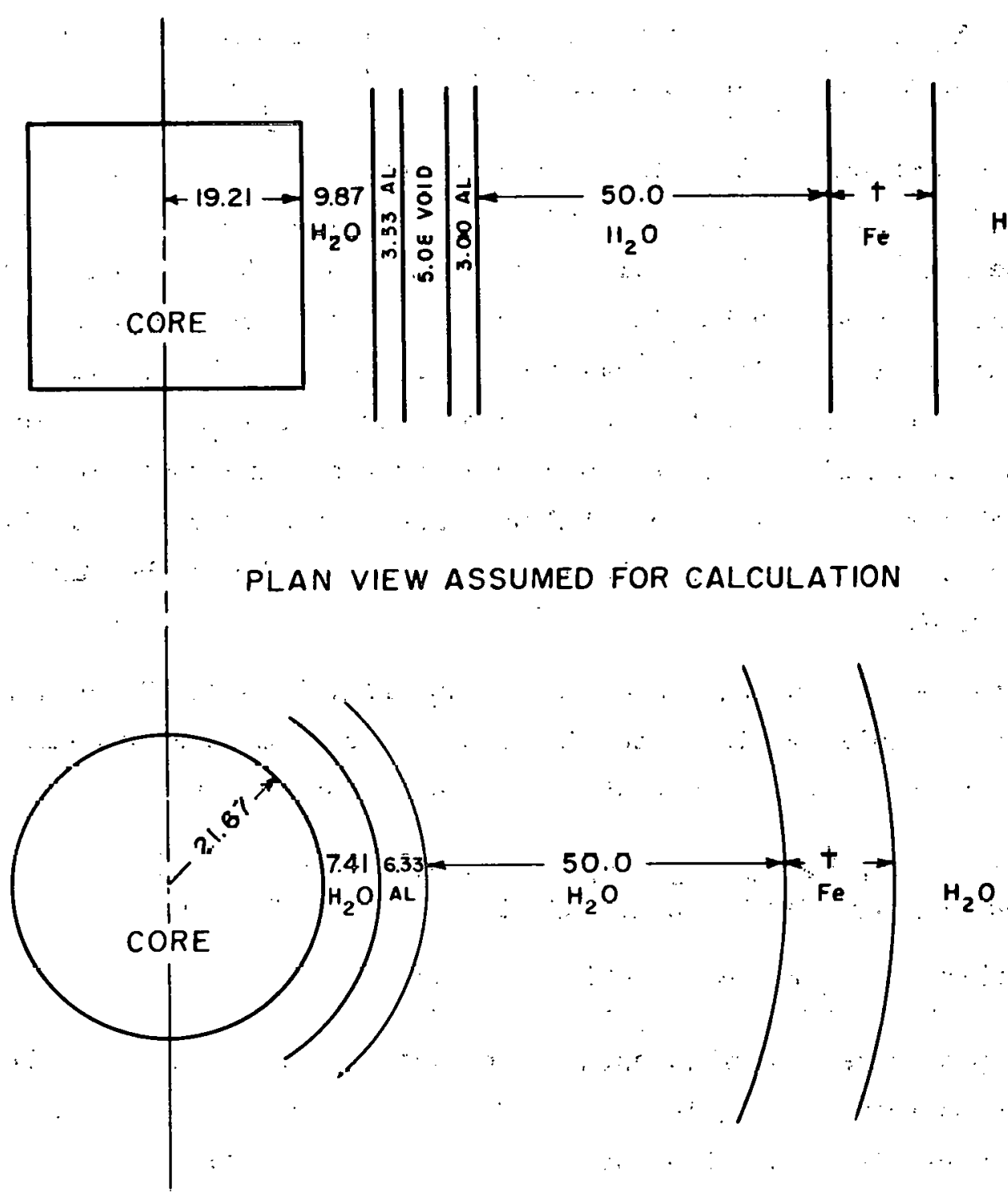

ALL DISTANCES IN CENTIMETERS

NOT TO SCALE

Figure 4 


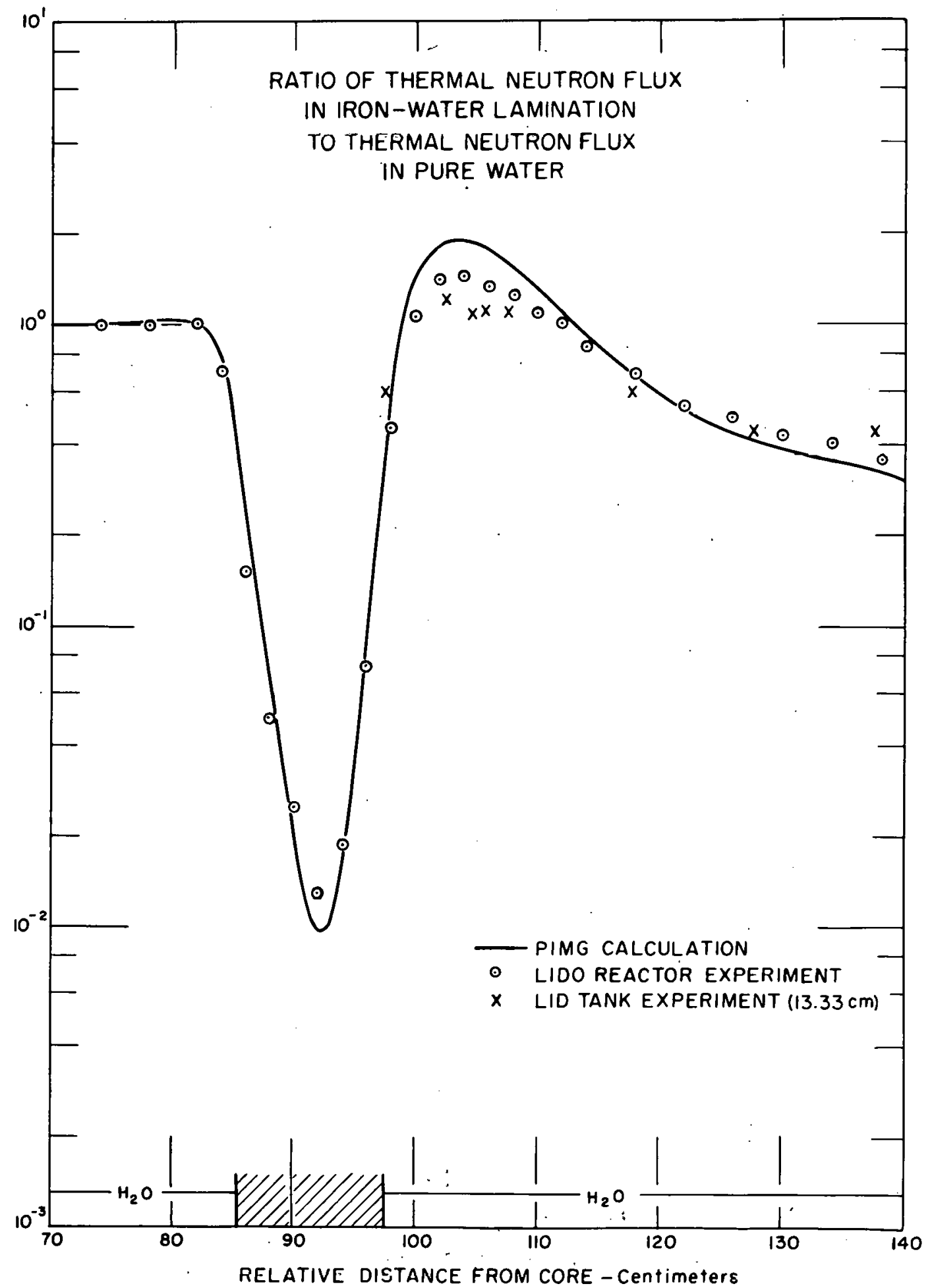

Figure 5 


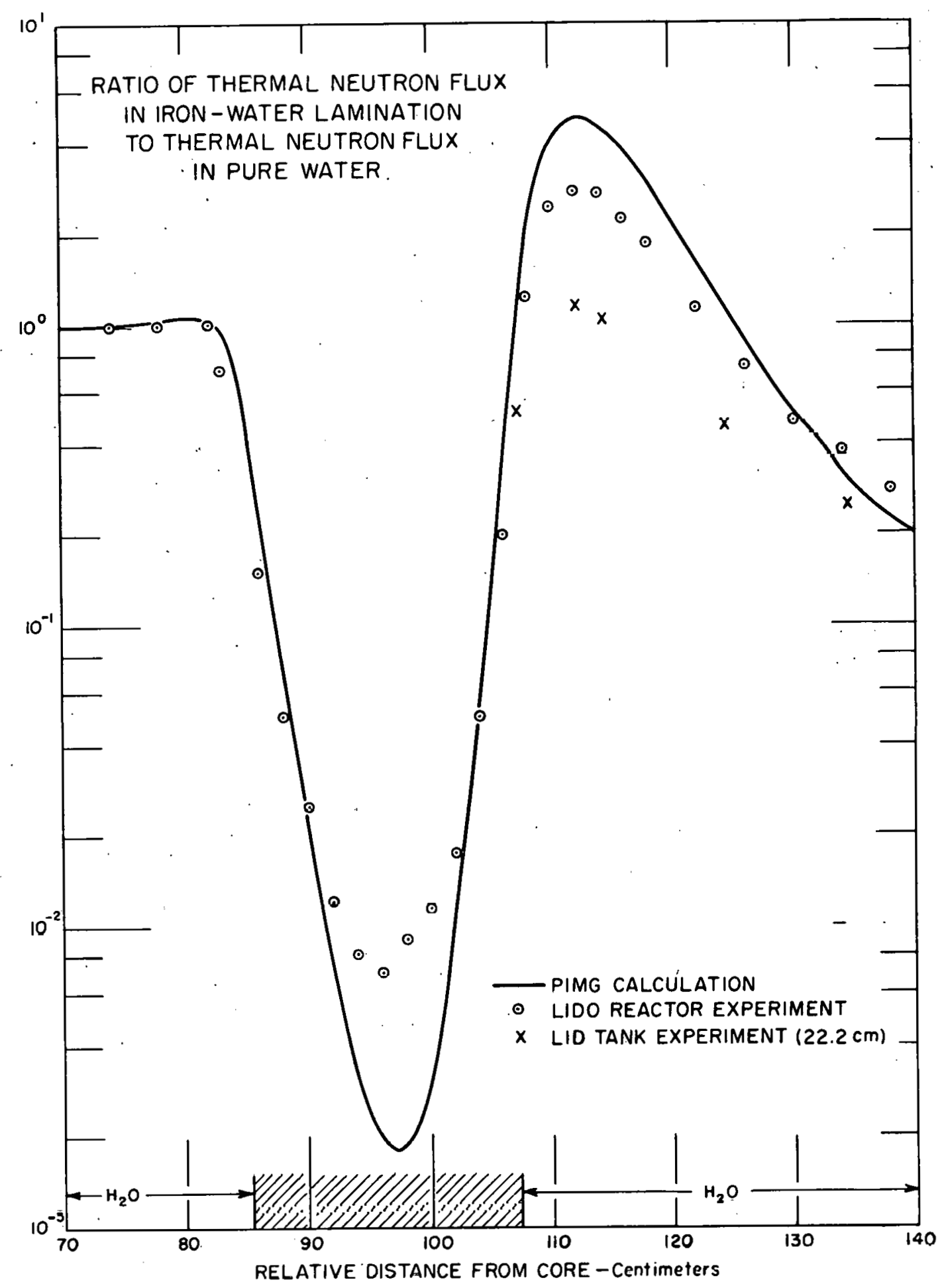

Figure 6 


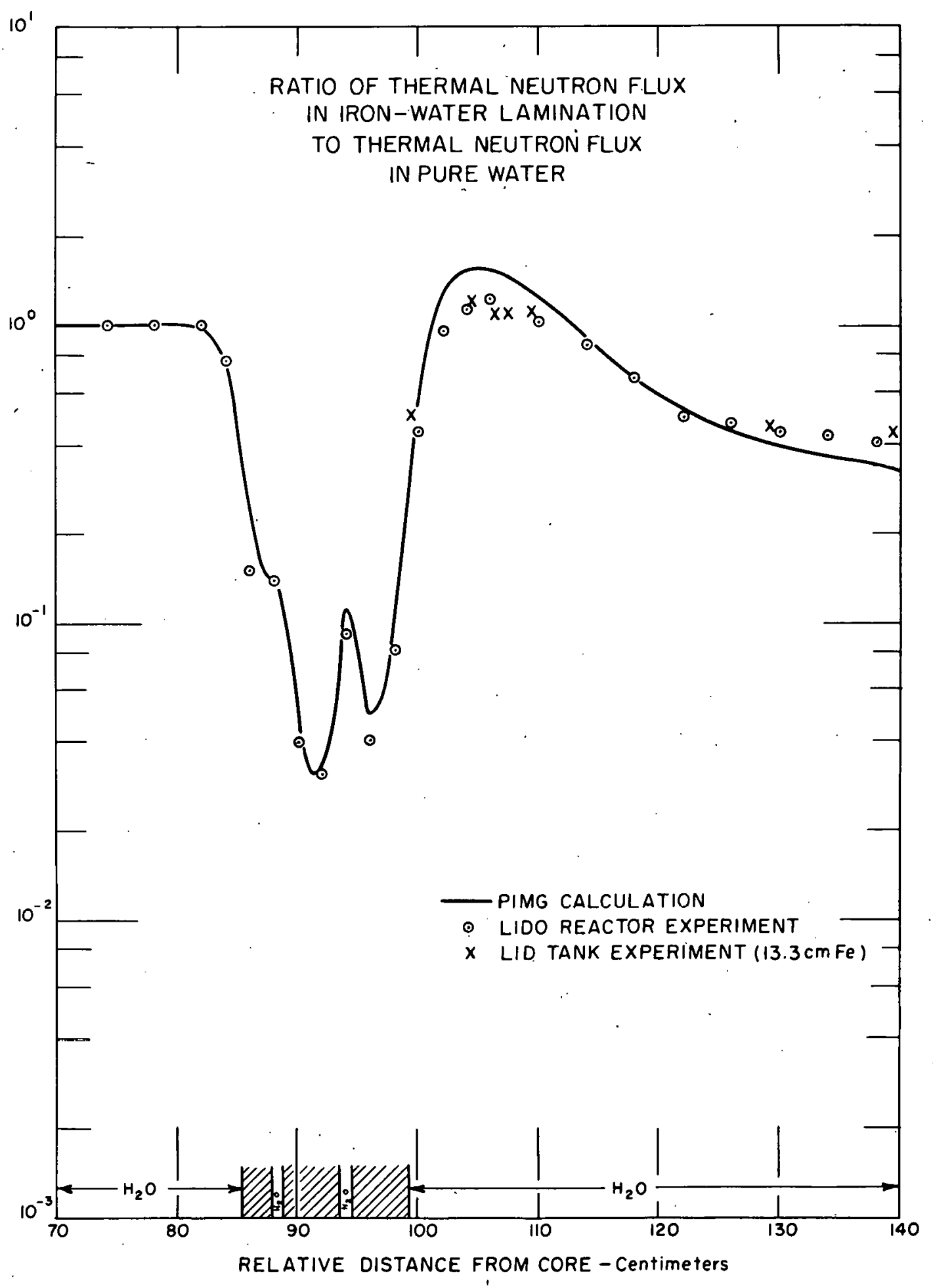

Figure 7 


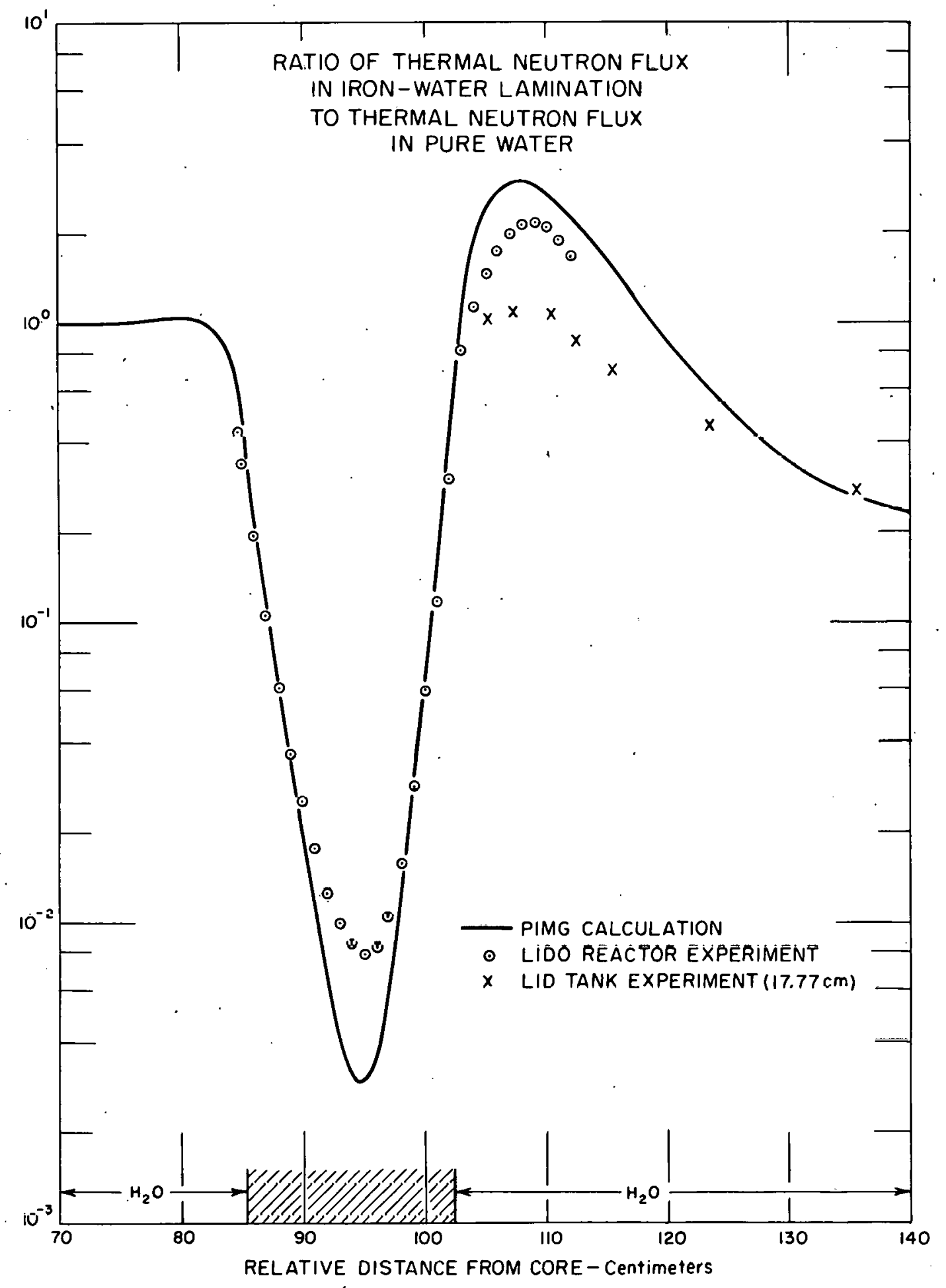

Figure 8 


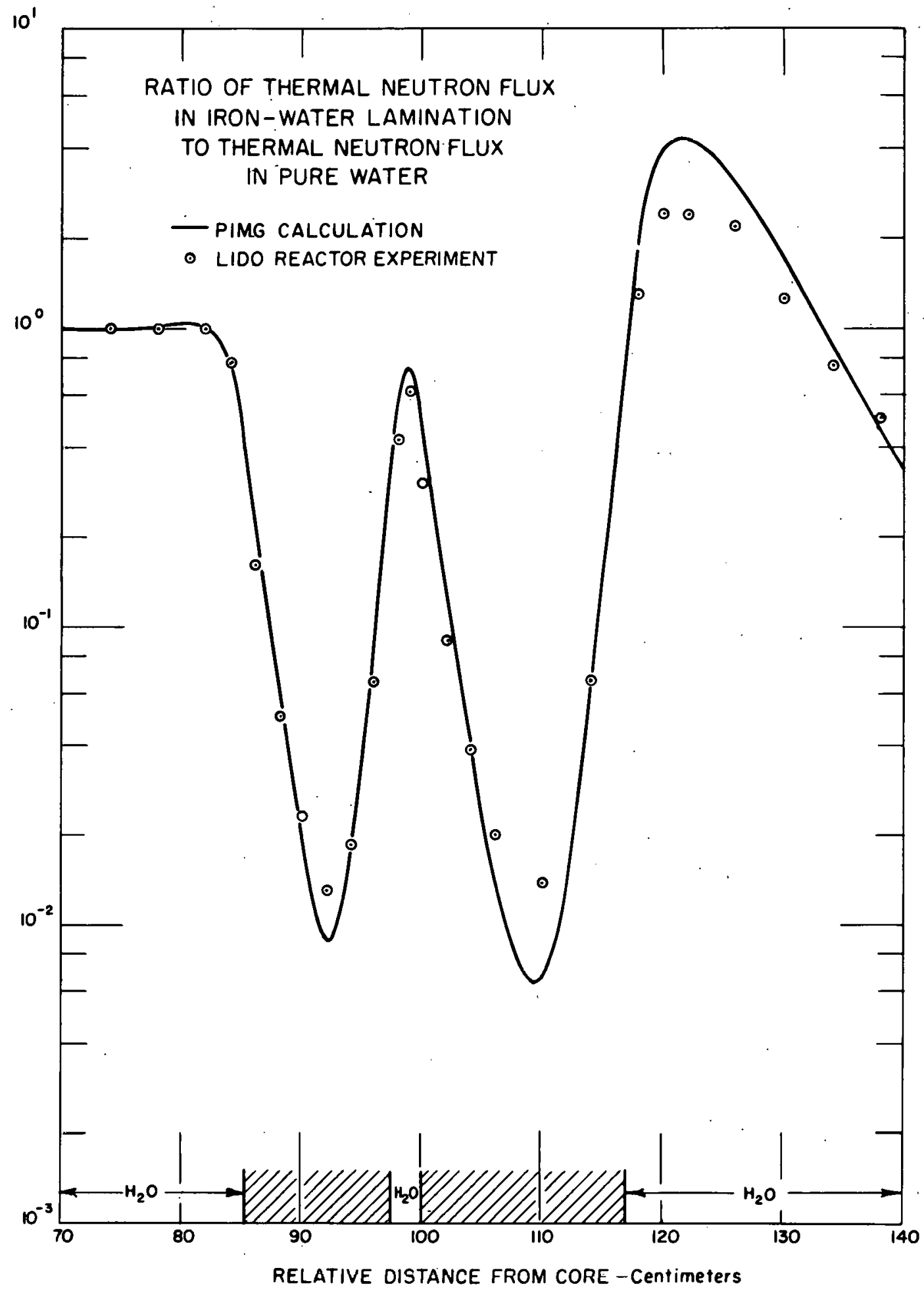

Figure 9 


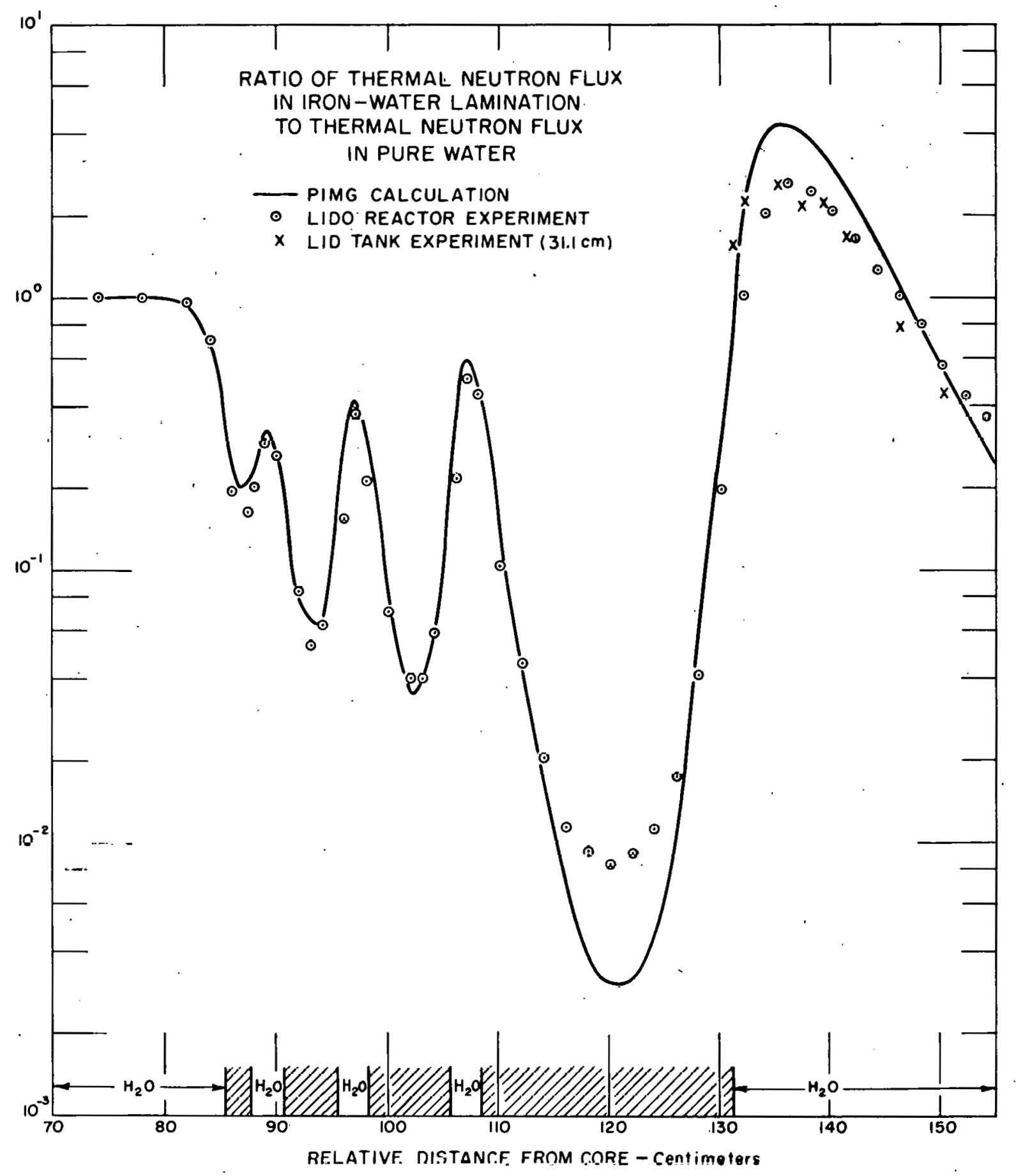

Figure 10 
seen the ratios calculated for the several configurations together with the geometry and the ratios obtained from the experiments. Also shown in these figures are the values of the ratio through approximately equivalent thicknesses of iron as obtained from ORNL Lid Tank Data (Ref 2). It is seen that calculations reproduce the observed LIDO ratios fairly well throughout these configurations. The calculational results are somewhat lower, than observed within a thick piece of iron. It should be noted, however, that in practical application, for example in the calculation of dose rates outside a shield from capture gamma-ray sources, the apparent underestimation of the flux within the iron has little effect on the over-all result sought.

B. Results for $\mathrm{Fe}-\mathrm{H}_{2} \mathrm{O}$ at the ORNL Lid Tank Facility

The ratio of the flux through three iron-water configurations to that in an all-water configuration has been obtained from experiment at the ORNL Lid Tank Shielding Facility (Ref 19). The results for one of these configurations is compared in Fig. 11 with a similar ratio using the results of PIMG calculations in slab geometry. The calculated results are seen to be in good agreement with the experimental results. Similar agreement has been noted for the other two configurations.

C. Results for Stainless Steel-Oil at the ORNL Lid Tank Facility

Thermal neutron fluxes have been measured in transformer oil following several stainless steel configurations in the ORNL Lid Tank Shielding Facility (Ref 20). The ratio of the flux measured with the presence of the stainless steel to that without for two of the configurations is seen in Figa 12 as a function of distance beyond the steel. PIMG calculations were run in slab geometry for these configurations. The calculations for the configuration containing $20 \mathrm{~cm}$ of oil following the source were run replacing the steel by iron whereas the calculations for the other configuration were run using stainless steel input information. The results, also seen in Fig. I2, indicate that the calculated ratios are somewhat greater than those determined from experiment. Also to be noted is that although one calculation uses stainless steel input information and one uses iron input information, the results when compared to experiment are not very sensitive to this change in this particular instance. Comparison between calculated and experimental ratios for several of the other configurations indicates even better agreement. 


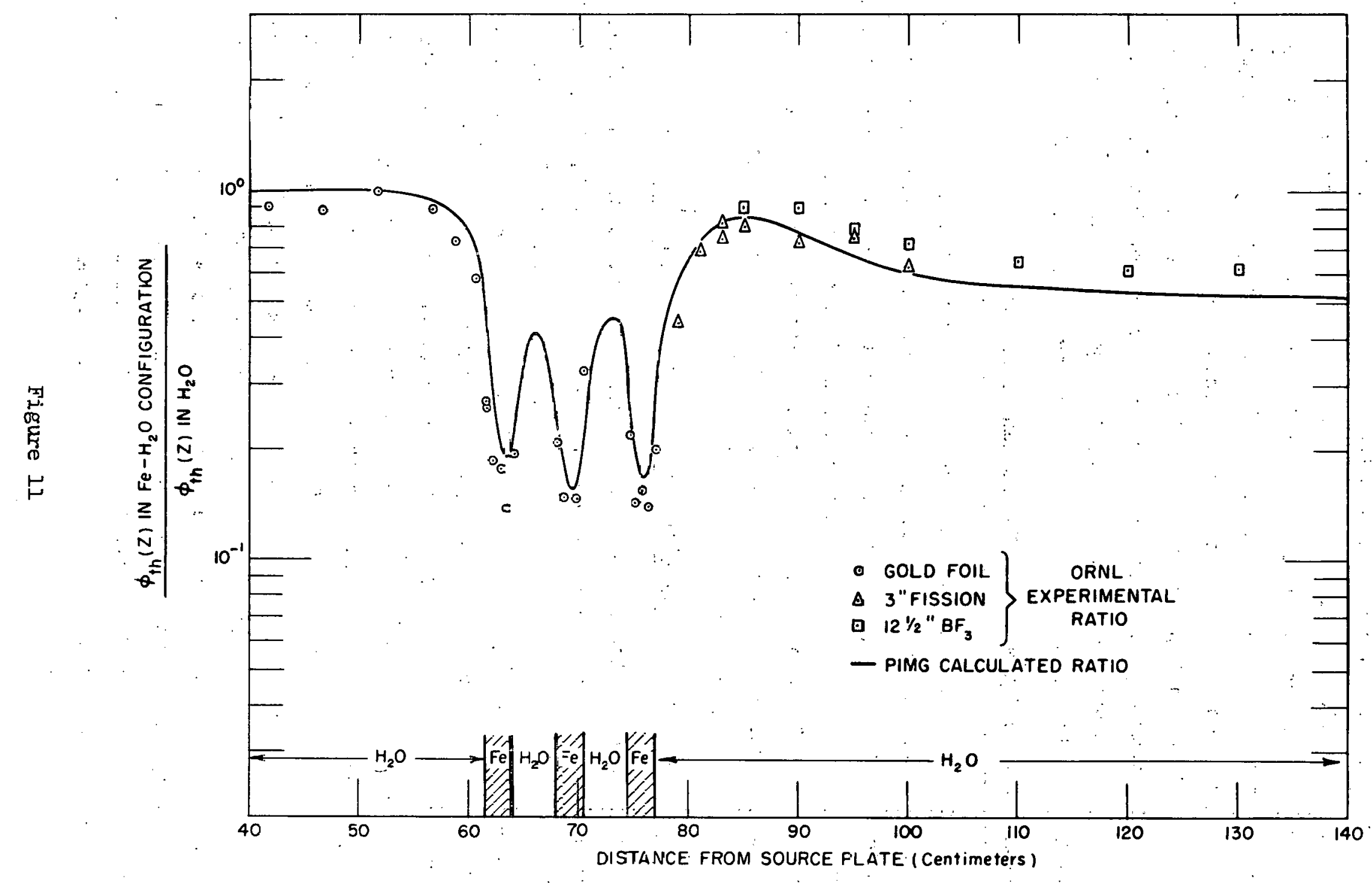




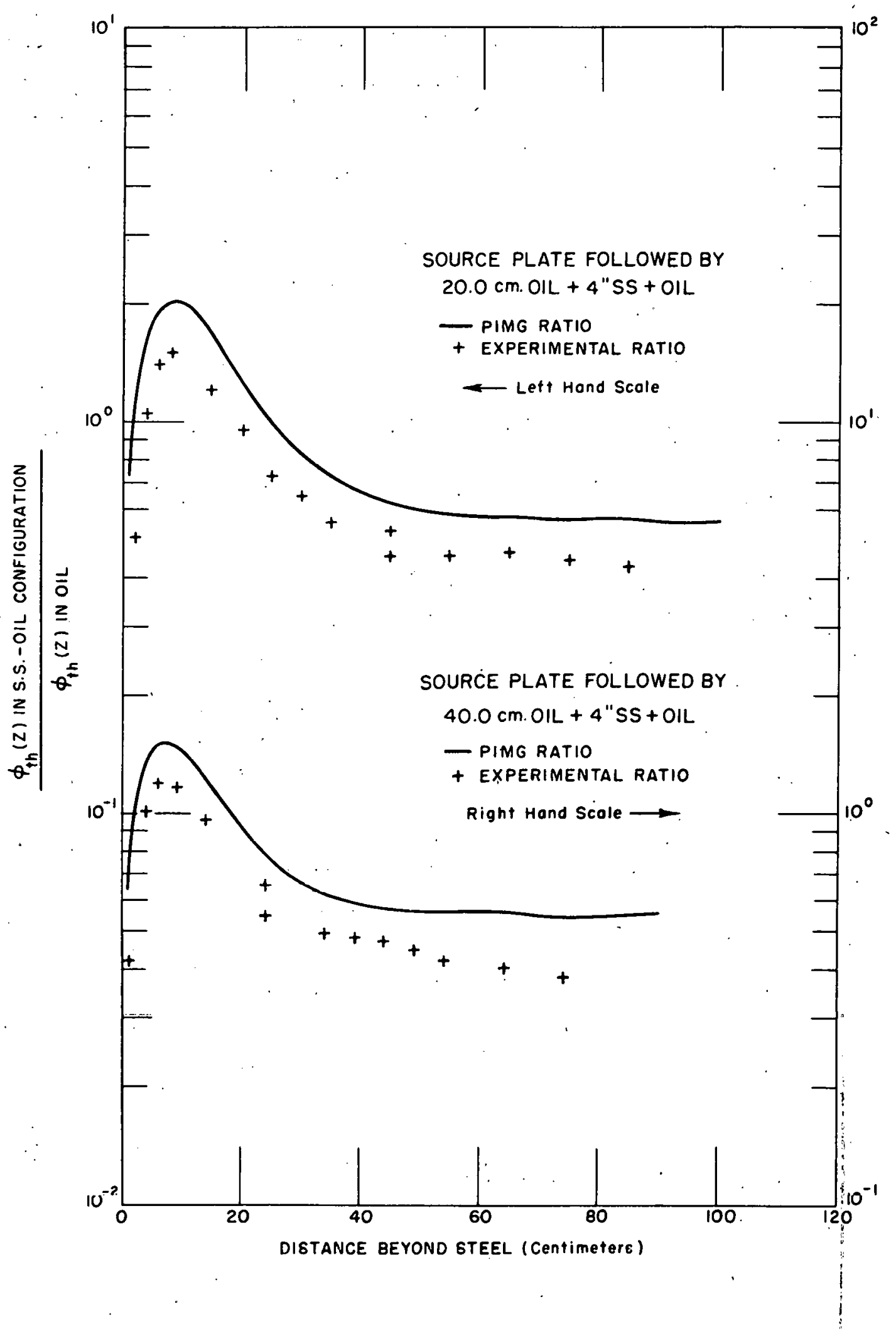

Figure 12 


\section{Results for Lead-Oil at the ORNL Lid Tank Facility}

Flux measurements have been made in transformer oil following several thicknesses of lead in the ORNL Lid Tank. Shielding Facility (Ref 21). The ratio of the flux measured with the presence of the lead to that without for two of the configurations is seen in Fig 13 as a function of distance beyond the lead. PIMG calculations were run in slab geometry for these configurations. The results, seen in Fig. 13, indicate that the calculated ratios reproduce the experimental ratios fairly well for a lead thickness of 4.5 in. and exceed the experimental ratio close to the thicker lead, slab in a manner similar to that observed for thick iron slabs (see Fig. 9).

\section{COMPARISON OF CALCULATION TECHNIQUE WITH EXPERIMENT IN AN IRON-WATER CONFIGURATION}

We now compare a calculation using the method presented here, with an experiment containing an iron-water shield surrounding the core. The source density distribution in the core is not well known. Measurements of dysprosium foil activities have been made in a primary shield mock-up surrounding a reactor in a critical facility and at one point in the core. The geometry of the region external to the core and relative fluxes are seen in Fig. 14. These experimental fluxes are relative foil activities normalized to a calculated thermal neutron flux in the core at a power of one watt in the reactor and are shown per fission neutron per unit volume per unit time in the ronre.

We have calculated the flux distribution through this assembly using the prescription described in this report. The source density distribution was assumed to be uniform throughout the volume of the core. The results are also seen in Fig. 14. It is seen that the prescription is in reasonable agreement with the shape of the experimental distribution. The magnitude comparison is difficul.t to make since the absolute source density distribution in the reactor was not measured and the fluxes were not obtained absolutely from the experiment. However, despite these difficulties, the magnitude agreement is good over the range investigated. 


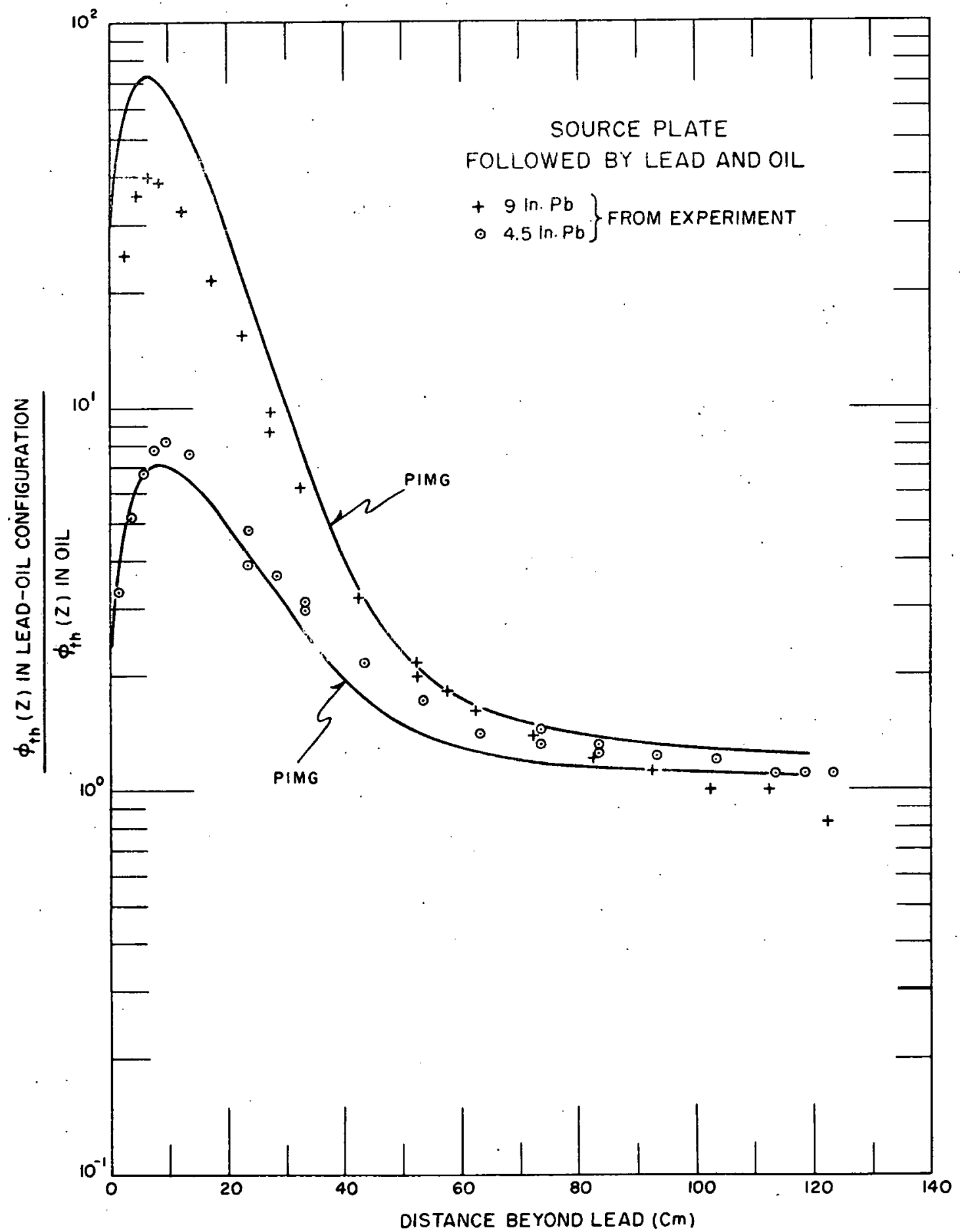

Figure 13 


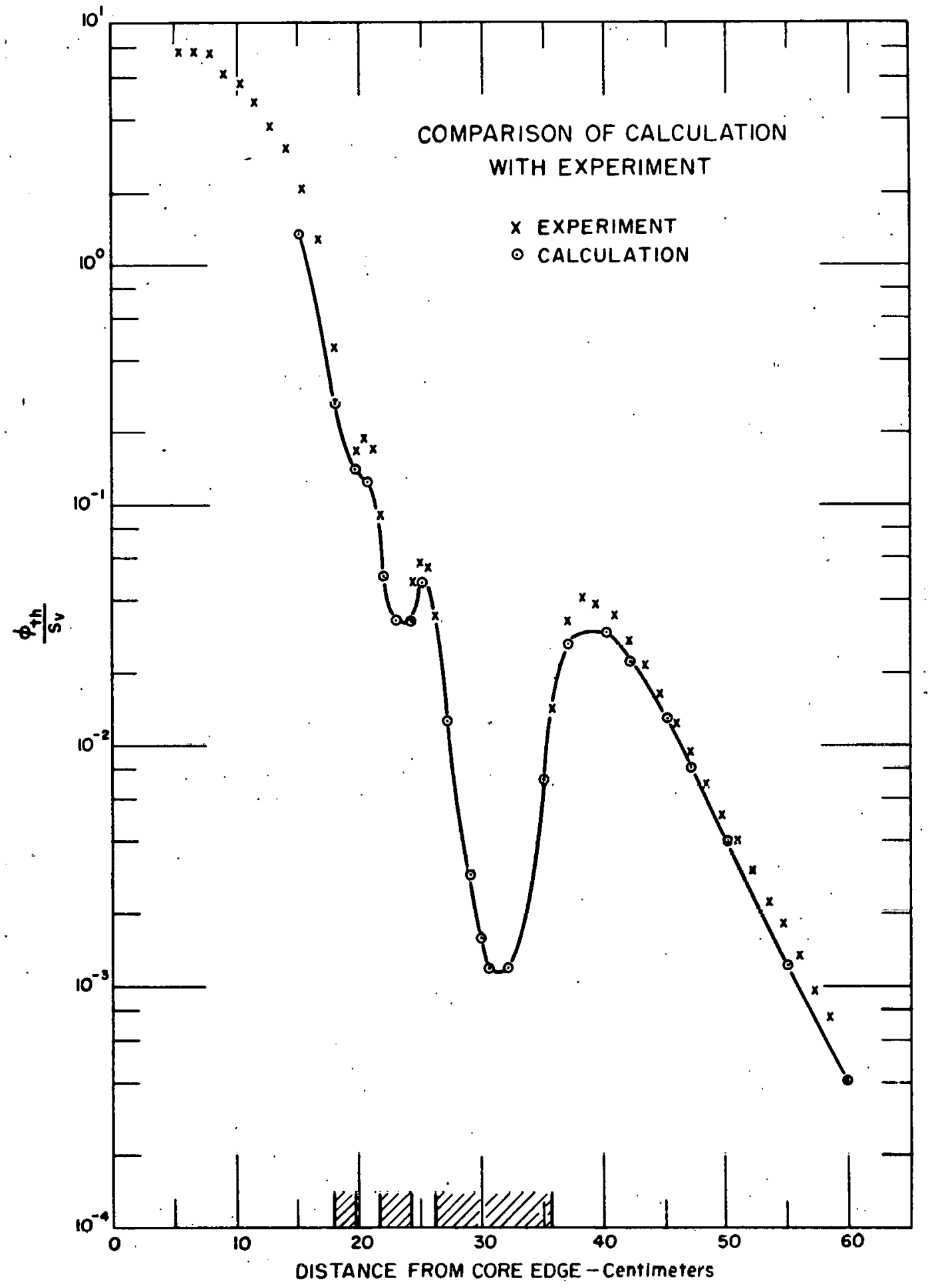

Figure 14 
VIII: EXTENSION TO TWO DIMENSIONS AND EPITHERMAL FLUXES

A. Two Dimensions

The multigroup diffusion code, PIMG, produces the spatial variation of each group flux in one dimension only. We have indicated how to adjust these results to obtain a more correct radial variation of the thermal neutron flux. There are several possible adjustments that can be made in Eq (I) to provide an estimate of the axial variation of the flux. The simplest method

is to replace the quantity $\phi_{W}^{P \cdot K} \cdot(r)$ by $\phi_{W}^{P \cdot K} \cdot(r, z)$ where the argument has a two-dimensional variation. This quantity can be obtained by integration over the spatial distribution of fission neutron sources, using a code such as SPIC-1. A more sophisticated method of estimating the axial variation would use results of two-dimensional, few-group codes such as PDQ (Ref 22) in place of PIMG results along with point kernel calculations. The group constants for each region in the two-dimensional, few-group code could be obtained from PIMG results as explained in Section VIII-B. The two-dimensional thermal neutron flux distribution would now be given by

$$
\phi_{t h}(r, z)=\frac{\Phi^{P D Q}(r, z) \phi_{W}^{P \cdot K} \cdot(r, z)}{\Phi_{W}^{P D Q}(r, z)}
$$

in which the quantities have definitionssimilar to those in Eq (1).

B. Estimation of Epithermal Fluxes

The capture rate of neutrons at epithermal energies can be estimated through the use of this prescription. Edits associated with the PIMG code are capable of providing few-group constants and fluxes. For example, one of these edits reduces the 55-group results to information for a fast or epithermal group and a thermal group. For this fast group, the edit provides spatial (pointwise) variation of such parameters as the diffusion constant, the absorption cross section, the group removal cross section, and the fast flux. In addition, regionwise variation of these parameters is available as well as regionwise group absorptions. Group constants for a region of given composition will vary depending on the location of the region with respect to the core. One can also obtain ratios of epithermal neutron fluxes and absorptions to the corresponding thermal neutron quantities. These ratios when 
multiplied by the thermal neutron flux or absorptions as determined by the prescription will provide estimates of the absolute epithermal fluxes or absorptions.

\section{ADEQUACY OF PIMG RESULTS}

The goal of this report is to present a method for calculating thermal neutron fluxes for shield design purposes. In the process of calculating these fluxes, additional spectral information is generated. In the previous section, we have indicated how few-group edits of the PIMG code could provide estimates of epithermal neutron fluxes and absorptions. In this section we would like to present a test of the adequacy of the additional spectral information so that limitations with respect to its use can be assessed.

If one presumes that the shape of the spectrum obtained from multigroup . calculations is the true shape from $10 \mathrm{Mev}$ down through thermal energies, one has the complete spectral as well as spatial variation of the neutron flux as a consequence of the use of this prescription. Thus one could calculate the fast neutron dose rate or other energy-dependent quantity in absolute magnitude. If one does not believe that the multigroup calculation results in the true spectral shape over the complete spectrum; it is possible to obtain averages of quantities over portions of the spectrum where the shape is presumed to be correct. For instance, it can be shown that the PIMG spectral shape above $0.3 \mathrm{Mev}$ in water is in reasonable agreement with moments method results (Ref 23). Thus a quantity such as the $R B E$ value for fast neutrons could be obtained from this spectrum and could be presumed to be a reasonable estimate in nonhydrogenous media as well if the microscopic information available for the PIMG code were adequate.

To test the adequacy of the complete multigroup spectral shape, we have compared results of fast neutron dose rate calculations using PIMG spectra with similar moments method results. A fast neutron dose rate kernel has been calculated from moments method results (Ref 23) and has been shown to agree. favorably with measurements in the Lid Tank Shielding Facility (Ref 12). We have determined the fast neutron dose rate in water at distances from 25 to $120 \mathrm{~cm}$ by integrating this kernel over the power density distribution in a core employing effective neutron removal cross sections for the nonhydrogenous materials of the core. We have used the identical technique to obtain the 
thermal neutron flux in water using Eq: (3). To this curve we have normalized the thermal neutron flux from PIMG using the renormalization procedure set forth in Section.II, and have obtained the fast neutron dose rate by applying to the fast neutron spectrum the same dose conversion factors that were used in Ref 23. A comparison is seen in Table $V$.

\section{TABLE V}

\begin{tabular}{ccc}
\hline & \multicolumn{2}{c}{ Fast Neutron Dose Rate - mrep/hr } \\
Distance from & \multicolumn{1}{c}{ Dose Kernel (Ref 23) } & $\frac{\text { PIMG }}{\text { Core Edge (cm) }}$ \\
\hline & $4.0 \times 10^{-4}$ & $4.8 \times 10^{-4}$ \\
25 & $4.7 \times 10^{-5}$ & $4.8 \times 10^{-5}$ \\
40 & $3.2 \times 10^{-6}$ & $2.8 \times 10^{-6}$ \\
60 & $2.9 \times 10^{-7}$ & $2.2 \times 10^{-7}$ \\
80 & $2.9 \times 10^{-8}$ & $2.1 \times 10^{-8}$ \\
100 & $3.2 \times 10^{-9}$ & $2.4 \times 10^{-9}$ \\
120 & & \\
\hline
\end{tabular}

Over the region investigated, the fast neutron dose rate calculated. using the technique presented herein is within $25 \%$ of the moments method results which are presumed to be correct. One can conclude that the PIMG spectral results in water are adequate and form a reasonable basị s of $\therefore$ approximation in nonhydrogenous media provided the microscopic information available is adequate. The spectral results can be used for estimating quantities that are not readily calculable using presently available. techniques.

\section{SUMMARY AND CONCLUSIONS}

We have described a method for calculating thermal neutron fluxes through primary shields which copes with heterogeneities and deep penetrations. The various portions of the method are arrived at theoretically utilizing microscopic nuclear information. Comparison with experiment of these various portions as well as the complete method is presented. Reliance on empirical information is minimized.

We have indicated how to obtain few-group fluxes, how to extend the results to two dimensions, and to what extent PIMG spectral results are reliable. 
APPENDIX: COMPARISON WITH OTHER PUBLISHED METHODS .

In this appendix we limit the discussion to a qualitative comparison of the methods for calculating thermal neutron fluxes presented by cooper (Ref 3 ), by Haffner (Ref 4), and the method presented herein:

The method that Cooper presents entails a calculation of the "removal" flux using the Albert-Welton point kernel (Ref 2) and effective removal cross sections (Ref 6). A three-group neutron diffusion model is employed, the three groups being the "removal" group, a slowing-down group for which neutrons scattered from the removal group form the source, and a thermal group. Some of the constants (mainly thermal constants) used in these equations are calculated from nuclear (microscopic) data, whereas others are determined by fitting the experimental results obtained in the Panel $\mathrm{C}$ Facility at the IIDO reactor. Also, in order that the magnitudes of the group fluxes be known, a starting point must be chosen such that equilibrium of the neutron spectrum is obtained. A question arises as to the availability of such a point in a typical design.

It is evident that this method relies heavily on empirical information. Therefore, it should predict fluxes fairly. well within the range of validity. defined by experiment.

Haffier (Ref 4) presents a method for colculating neutron spertra in shields. This method predicts flux distributions utilizing a combination of a point-to-point attenuation kernel and a multigroup $P$ or $\dot{S}$ approximation calculation as used in reactor analysis. The multigroup results are prasimen to give the correct neutron spectral variation but an incorrect spatial. variation. The point-to-point attenuation kernel is supposed to reproduce the fast neutron attenuation and thereby is used to correct the spatial variation. It is indicated that the point kernel can be the Albert-Welton kernel, although any method which is adequate for computing fast neutron dose rate (or flux) would work.

Mathematically, Haffner presents his prescription in the following equation:

$$
\Phi(E, r)=\frac{D(r) \Phi(E, r)}{\int G_{1}(E) \Phi(E, r) d E}
$$


where $\Phi(E, r)$ normalized spectrum as a function of energy and position,

$\Phi .(E, r)$ unnormalized spectrum calculated by $P$ or $S$ approximations,

$D(r)$ " fast neutron dose rate or flux as calculated by the point kernel,

$G_{1}(E)$ response function used to measure $D(r)$. Thus, if $D(r)$ is the fast neutron dose rate, $G_{1}(E)$ is the energy dependence of the fast neutron dosimeter.

Both $D(r)$ and $\Phi(E, r)$ are determined in the exact geometry of the problem. Thus, these functions are to be calculated within portions of the shield whether these regions be hydrogenous or nonhydrogenous. Effective removal cross sections (Ref 6) are used for the nonhydrogenous materials.

Although the technique for calculating the neutron energy spectrum appears to compare favorably with experiment, as presented by Haffner, we believe the following, to be areas of possible inadequacy in predicting thermal neutron fluxes:

a) Assumption that the energy dependence of the complete neutron spectrum from $10 \mathrm{Mev}$ down to thermal energies is accurately given by the results of the $\mathrm{P}$ or $\mathrm{S}$ approximations.

b) Use of effective removal cross sections to predict fast neutron fluxes or dose rates within a nonhydrogenous medium.

c) Use of the Albert-Welton kernel for fast neutron flux or dose rate calculations at all distances from fission neutron sources in hydrogenous media.

The method presented in this report resembles that presented by Haffner. However, the following should be noted.

The point kernel that is to be used in the calculation of the spatial correction factor is derived for use in a homogeneous medium and should be applicable using effective removal cross sections in the heterogeneous system described (core surrounded by water) within the limitations specified in Section II. The point kernel that Haffner suggests using is also applicable to homogeneous media calculations. However, its applicability to all regions in a heterogeneous shield using effective removal cross sections has never been justified.

The correction factor herein calculated characterizes the thermal energy region. Thus one does not have to make explicit assumptions about the shape of the spectrum generated by a multigroup calculation. 


\section{ACKNOWLEDGEMENT}

The authors wish to acknowledge the assistance of Miss Jane Simcic in performing most of the hand calculations and in processing the many digital calculations that were employed in the formulation of the technique reported herein.

\section{REFERENCES}

1. "Reactor Shielding Design Manual," TID-7004, March 1956.

2. Albert and Welton, "A Simplified Theory of Neutron Attenuation and Its Application to Reactor Shield Design," WAPD-15 (DeI), November 1950.

3. Cooper, Jones, and Horton, "Some Design Criteria for Hydrogen-Metal Reactor Shields," A/Conf. 15/P/84, May 1958.

4. J. W. Haffner, "Neutron Energy Spectrum Calculations in Reactor shields;" Preprint V-84 to Nuclear Engineering and Science Conference, April 1959.*

5. C. H. Westcott, Journal of Nuclear Energy 2, 59 (1955).

6. Chapman and Storrs, "Effective Neutron Removal Cross Sections for Shielding," AECD-3978, September 1955.

7. Bohl, Gelbard, Ryan, and Fike, "PIMG - A One-Dimensional Multigroup $\mathrm{P}_{1}$ Code for the IBM-704," WAPD-TM-135, July 1959.

8. H. Amster, "Cross Sections in the SOFOCATE Code: Second Deck," WAPD-TM-67, June 1957.

9. Bohl, Belbard, and Ryan, "MÜFT-4 Fast Neutron Spectrum Code for the IBM-104," WAPD-TM-72, July 1957.

10. P. A. Gillis, "SPIC-1 - An IBM-704 Code to Calculate the Neutron Distribution Outside a Right Circular Cylindrical Source," WAPD-TM-196, November 1959.

11. Certaine and Aronson, "Distribution of Fission Neutrons in Water at the Indium Resonance Energy,". NDA-15C-40, June 1954.

12. H. Goldstein, Fundamental Aspects of Reactor Shielding, Addison-Wesley Publishing $\mathrm{Co}_{0}$, Inc., Reading, Mass., 1959.

13. Hill, Roberts, and Fitch, Journal of Applied Physics 26, 1013 (1955).

14. "Applied Nuclear Physics Division Annual Progress Report for the period ending September 1, 1957," ORNL-2389, Paper 9.2, Novemher 1957.

15. Ibid, Paper 8.10. 
16. "Attenuation in Water of Radiation from the Bulk Shielding Reactor," ORNL-2518, July 1958.

17. E. B. Johnson, "Power Calibration for BSR Loading 33," ORNL-CF-11-30, November 1957 .

18. Private Communication from C. Cooper to K. Shure, August 18, 1959.

19. Private Communication from F. Haag to K. Shure, April 16, 1959.

20. Private Communication from W. Zobel to K. Shure, September 19, 1958.

21. "Neutron Physics Division Annual Progress Report for the period ending September 1,. 1958," ORNL-2609, Paper 7.4, October 1958.

22. Bilodeau, Cadwell, Dorsey, Fairey, and Varga, "PDQ - AN IBM-704 Code to Solve the Two-Dimensional Few-Group Neutron-Diffusion Equations," WAPD-TM-70, August 1957.

23. Aronson, Certaine, Goldstein, and Preiser, "Penetration of Neutrons from a Point Isotropic Fission Source in Water," NY0-6267, September 1954. 